\title{
STEADY PERIODIC WATER WAVES WITH CONSTANT VORTICITY: REGULARITY AND LOCAL BIFURCATION
}

\author{
ADRIAN CONSTANTIN AND EUGEN VARVARUCA
}

\begin{abstract}
This paper studies periodic traveling gravity waves at the free surface of water in a flow of constant vorticity over a flat bed. Using conformal mappings the free-boundary problem is transformed into a quasilinear pseudodifferential equation for a periodic function of one variable. The new formulation leads to a regularity result and, by use of bifurcation theory, to the existence of waves of small amplitude even in the presence of stagnation points in the flow.
\end{abstract}

Keywords and Phrases: water waves, vorticity, conformal mapping, Hilbert transform. AMS Mathematics Subject Classification (2000): 76B15, 35J25, 30E25, 35Q35, 35R35.

\section{INTRODUCTION}

The problem of spatially periodic traveling gravity water waves with constant vorticity $\gamma$ on a flow of finite depth can be formulated as the free-boundary problem of finding

- a domain $\Omega$ in $(X, Y)$-plane, whose boundary consists of the real axis

$$
\mathcal{B}=\{(X, 0): X \in \mathbb{R}\},
$$

representing the flat impermeable water bed, and an a priori unknown curve expressed in parametric form as

$$
\mathcal{S}=\{(u(s), v(s)): s \in \mathbb{R}\},
$$

with

$$
u(s+L)=u(s)+L, \quad v(s+L)=v(s) \quad \text { for all } s \in \mathbb{R},
$$

representing the free surface of the water, which is $L$-periodic in the horizontal direction;

- a function $(X, Y) \mapsto \psi(X, Y)$ which is $L$-periodic in $X$ throughout $\Omega$, representing the stream function giving the velocity field $\left(\psi_{Y},-\psi_{X}\right)$ in a frame moving at the constant wave speed, which satisfies the following equations and boundary conditions:

$$
\begin{aligned}
& \Delta \psi=-\gamma \quad \text { in } \Omega, \\
& \psi=-m \quad \text { on } \mathcal{B}, \\
& \psi=0 \quad \text { on } \mathcal{S}, \\
& |\nabla \psi|^{2}+2 g Y=Q \quad \text { on } \mathcal{S} .
\end{aligned}
$$

Here $g$ is the gravitational constant of acceleration, the constant $m$ is the relative mass flux, while the constant $Q$ is related to the hydraulic head (see the discussion in [12]). The level sets of $\psi$ are the streamlines, while a point where the gradient of $\psi$ vanishes is called a stagnation point.

We are interested in the existence, regularity and geometric properties of solutions of (1.1). The physical motivation for our study lies in the desire to understand wave-current interactions, since nonzero vorticity is the hallmark of a non-uniform underlying current [10, 12. Even in the simplest case of a linear current, which corresponds to a constant vorticity $\gamma$ in (1.1), numerical studies [16, 22, 23, 27] point 
to the existence of large-amplitude waves with overhanging profiles and critical layers (regions in the fluid bounded by closed streamlines and containing stagnation points). This is in striking contrast to the extensively studied irrotational case [7, 31, 36], for which any wave profile is necessarily the graph of a function and there can be no critical layers in the fluid [37, 40, 41]. The assumption of an underlying current with nonzero constant vorticity (or linear shear) provides us with the simplest case of a flow that is not irrotational and is attractive for analytical tractability. However, this setting is not a mere mathematical convenience, being physically relevant. Indeed, on areas of the continental shelf and in many coastal inlets the most significant currents are the tides and tidal flows are the most regular and predictable currents [19, 33], considered to be two-dimensional flows of constant vorticity: negative constant vorticity is generated when the flow of the current is directed towards the shore (flood) and positive constant vorticity when the current is directed back out to sea (ebb) [16, 45].

This paper addresses some of the mathematical challenges posed by the study of solutions of (1.1) with overhanging profiles or critical layers. In all previous studies of existence of solutions of (1.1), equivalent formulations of the problem over a fixed domain are essential. Bifurcation theory is then the most successful tool used for existence results. For irrotational flows $(\gamma=0$, representing uniform flows without vorticity), a hodograph transformation maps the unknown domain occupied by the water into a fixed horizontal strip in a complex plane where the variable is the complex potential of the fluid flow. In these coordinates, the classical approach is to reformulate (1.1) as a nonlinear singular integral equation for a function of one variable which gives the angle of inclination between the tangent to the free surface and the horizontal [1, 20, 36]. A more recent approach [5, 6, 31] (for the analogous problem of waves of infinite depth) uses a reformulation of the problem as a pseudodifferential equation for a function of one variable which gives the elevation of the free surface when the fluid domain is the conformal image of a strip [2].

In this paper we show that the second approach can be extended to the case of any constant vorticity $\gamma$. The basic idea is to seek the fluid domain as the conformal image of a strip, regardless of the unavailability of a complex potential. The new formulation is presented in Section 2. It imposes no restriction on the geometry of the free surface or on the streamline pattern, and in particular it can handle overhanging profiles and critical layers. In order to avoid distracting technicalities, we assume at the outset a modest amount of regularity of the free surface, namely $C^{1, \alpha}$ for some $\alpha \in(0,1)$, though arguing along the lines of [31, 41] one could verify the equivalence of the formulations also in the framework of weak solutions [43. As the new formulation involves the periodic Dirichlet-Neumann operator and the periodic Hilbert transform associated to a strip, Section 3 studies some useful properties of these operators. Section 4 is devoted to regularity of solutions of the new equation, showing in particular that any $C^{1, \alpha}$ free surface with no stagnation points is actually $C^{\infty}$. In Section 5 we prove the existence of waves of small amplitude using bifurcation theory. The analysis reveals the existence of such waves with a critical layer, a fact first proved rigorously only very recently in [44, though anticipated by a formal argument of Kelvin [34, who presented the famous cat's eye picture of the streamline pattern.

Before proceeding with the bulk of the paper we would like to comment upon the relation to some recent investigations on water waves with vorticity. For general vorticity but under the assumption that $\psi$ is monotone in the vertical direction, a hodograph transform $(X, Y) \mapsto(X, \psi)$ was used in [12] to transform (1.1) into a nonlinear boundary value problem for a quasilinear elliptic equation in a strip, which permits the investigation of waves of small and large amplitude (see also [9, 13, 42, 43]). Thus 
the formulation in [12] cannot describe neither critical layers nor overhanging profiles. The recent paper [44 deals with waves of small amplitude in a flow of constant vorticity which could contain stagnation points. The approach of [44] cannot handle overhanging profiles. For a free surface of the form $\mathcal{S}=\{(X, \eta(X)): X \in \mathbb{R}\}$, it reformulates (1.1) as a nonlinear boundary problem for an elliptic equation in a strip by performing the flattening change of variables $(X, Y) \mapsto(X, ¥)=\left(X, \frac{Y}{\eta(X)}\right)$. The free surface is thus transformed into the horizontal line $¥=1$ and, in the new variables, one has to solve a system of equations for a function of two variables and one of one variable. Our analysis of small amplitude waves is much simpler than that in [44. The new formulation, which has an elegant structure, opens up the possibility of using global bifurcation theory to prove the existence of waves of large amplitude with critical layers and/or overhanging profiles, a prospect which seems daunting for the formulation in [44. In addition, our approach has the advantage that in the reformulation we do not depart from harmonic function theory, and the fine structural properties associated with harmonic functions continue to be relevant. Such properties made it possible to describe the particle motion beneath irrotational waves [8, 14] and the exploration of this aspect for flows with constant vorticity is now plausible.

\section{ReFormulation of the FREE-BOUNDARY PROBLEM}

In this section we present the reformulation of the free-boundary problem (1.1) as the quasilinear pseudodifferential equation (2.9) for a periodic function of one variable. This involves the periodic Dirichlet-Neumann operator and the periodic Hilbert transform for a strip, so we start with a discussion of the definitions and basic properties of these operators.

For any integer $p \geq 0$ and $\alpha \in(0,1)$ we denote by $C^{p, \alpha}$ the standard space of functions whose partial derivatives up to order $p$ are Hölder continuous with exponent $\alpha$ over their domain of definition. By $C_{\text {loc }}^{p, \alpha}$ we denote the set of functions of class $C^{p, \alpha}$ over any compact subset of their domain of definition. We will need the following local version of Privalov's Theorem [24, Chapter V, §E, p. 100] (see [39, Lemma 2.2] for the proof of a very similar result). A more refined, global version of Privalov's Theorem, related to but different from that in [24], will be proved in Appendix B.

Lemma 2.1. For any $t>0$, we denote $\mathbb{D}_{t}^{+}=\left\{(x, y) \in \mathbb{R}^{2}: x^{2}+y^{2}<t^{2}, y>0\right\}$. Let $r>0$ and $Z+i W$ be a holomorphic function in $\mathbb{D}_{r}^{+}$, where $Z$ and $W$ are real-valued functions. Suppose that $W$ is continuous in $\mathbb{D}_{r}^{+} \cup\{(x, 0): x \in(-r, r)\}$, and let

$$
w(x)=W(x, 0), \quad x \in(-r, r) .
$$

If $w \in C_{\mathrm{loc}}^{p, \alpha}((-r, r))$ for some integer $p \geq 0$ and $\alpha \in(0,1)$, then $W$ and $Z$ are of class $C^{p, \alpha}$ in the closure of $\mathbb{D}_{t}^{+}\left(x_{0}\right)$ for every $t \in(0, r)$.

For any $d>0$, let $\mathcal{R}_{d}$ be the strip

$$
\mathcal{R}_{d}=\left\{(x, y) \in \mathbb{R}^{2}:-d<y<0\right\} .
$$

For any integer $p \geq 0$ and $\alpha \in(0,1)$ we denote by $C_{2 \pi}^{p, \alpha}$ the space of functions of one real variable which are $2 \pi$-periodic and of class $C^{p, \alpha}$. For any $w \in C_{2 \pi}^{p, \alpha}$, let $W \in C^{p, \alpha}\left(\overline{\mathcal{R}}_{d}\right)$ be the unique solution of

$$
\begin{aligned}
& \Delta W=0 \quad \text { in } \mathcal{R}_{d}, \\
& W(x,-d)=0, \quad x \in \mathbb{R}, \\
& W(x, 0)=w(x), \quad x \in \mathbb{R} .
\end{aligned}
$$


The function $(x, y) \mapsto W(x, y)$ is $2 \pi$-periodic in $x$ throughout $\mathcal{R}_{d}$. For $p \geq 1$ integer, we define $\mathcal{G}_{d}(w)$ by

$$
\mathcal{G}_{d}(w)(x)=W_{y}(x, 0), \quad x \in \mathbb{R} .
$$

(Throughout the paper, the subscripts $x, y, X, Y$ denote partial derivatives, and are the only subscripts with this meaning.) Then $\mathcal{G}_{d}(w) \in C_{2 \pi}^{p-1, \alpha}$. The mapping $w \mapsto \mathcal{G}_{d}(w)$ is called the periodic Dirichlet-Neumann operator for a strip, and is a (bounded) linear operator from $C_{2 \pi}^{p, \alpha}$ to $C_{2 \pi}^{p-1, \alpha}$. Note in particular that if $w$ is a constant function taking the value $c$, then

$$
W(x, y)=\frac{c}{d}(y+d), \quad(x, y) \in \mathcal{R}_{d},
$$

and hence

$$
\mathcal{G}_{d}(c)=c / d
$$

Suppose now that $Z$ is a harmonic function in $\mathcal{R}_{d}$, uniquely determined up to a constant, such that $Z+i W$ is holomorphic in $\mathcal{R}_{d}$. By Lemma 2.1, $Z \in C^{p, \alpha}\left(\overline{\mathcal{R}}_{d}\right)$. Note that the function

$$
(x, y) \mapsto Z(x+2 \pi, y)-Z(x, y)
$$

is constant in $\mathcal{R}_{d}$, as can be seen by taking its partial derivatives and using the Cauchy-Riemann equations. Let $K \in \mathbb{R}$ be such that

$$
Z(x+2 \pi, y)-Z(x, y)=K, \quad(x, y) \in \mathcal{R}_{d} .
$$

For every $x \in \mathbb{R}$ and $y \in(-d, 0)$, it follows using the Cauchy-Riemann equations that

$$
\begin{array}{r}
\frac{d}{d y} \int_{x}^{x+2 \pi} W(t, y) d t=\int_{x}^{x+2 \pi} W_{y}(t, y) d t \\
=\int_{x}^{x+2 \pi} Z_{x}(t, y) d t=Z(x+2 \pi, y)-Z(x, y)=K .
\end{array}
$$

Integrating the above relation from $y=-d$ to $y=0$, and taking into account the boundary conditions satisfied by $W$ in (2.1), it follows that

$$
K=\frac{2 \pi[w]}{d}
$$

where $[w]$ denotes the average of $w$ over one period. Hence $K=0$ if and only if $[w]=0$. For any integer $p \geq 0$ and $\alpha \in(0,1)$, let us denote by $C_{2 \pi, \circ}^{p, \alpha}$ the class of functions in $C_{2 \pi}^{p, \alpha}$ which have zero mean over one period.

Let $w \in C_{2 \pi, \alpha}^{p, \alpha}$. Then the function $(x, y) \mapsto Z(x, y)$ is $2 \pi$-periodic in $x$ throughout $\mathcal{R}_{d}$. We normalize the constant in the definition of $Z$ by the requirement that $x \mapsto$ $Z(x, 0)$, which is a $2 \pi$-periodic function, has zero mean over one period. We define $\mathcal{C}_{d}(w)$ by

$$
\mathcal{C}_{d}(w)(x)=Z(x, 0), \quad x \in \mathbb{R} .
$$

The mapping $w \mapsto \mathcal{C}_{d}(w)$ is called the periodic Hilbert transform for a strip, and is a (bounded) linear operator from $C_{2 \pi, o}^{p, \alpha}$ into itself. It is easy to see from the definitions and the Cauchy-Riemann equations that, when $w \in C_{2 \pi, o}^{p, \alpha}$ for $p \geq 1$ integer,

$$
\mathcal{G}_{d}(w)=\left(\mathcal{C}_{d}(w)\right)^{\prime}=\mathcal{C}_{d}\left(w^{\prime}\right)
$$

A more detailed account of the properties of the operators $\mathcal{G}_{d}$ and $\mathcal{C}_{d}$ is given in Section 3. Note that the space of $2 \pi$-periodic harmonic/holomorphic functions in a strip can be identified with the space of harmonic/holomorphic functions in an annulus, by means of an explicit conformal mapping, and thus the operators $\mathcal{G}_{d}$ and $\mathcal{C}_{d}$ can be equivalently defined in the setting of an annulus. 
Suppose now that $w \in C_{2 \pi}^{p, \alpha}$. By (2.2), (2.3) and (2.5), one can write

$$
Z+i W=\frac{[w]}{d}(x+i(y+d))+Z_{0}+i W_{0} \quad \text { in } \mathcal{R}_{d},
$$

where $Z_{0}, W_{0} \in C^{p, \alpha}\left(\overline{\mathcal{R}}_{d}\right)$ are $2 \pi$-periodic in $x$ throughout $\mathcal{R}_{d}$ and

$$
\begin{aligned}
& \Delta W_{0}=0 \quad \text { in } \mathcal{R}_{d}, \\
& W_{0}(x,-d)=0, \quad x \in \mathbb{R}, \quad \\
& W_{0}(x, 0)=w(x)-[w], \quad x \in \mathbb{R} .
\end{aligned}
$$

We normalize the constant in the definition of $Z$ by the requirement that $x \mapsto Z_{0}(x, 0)$, which is a $2 \pi$-periodic function, has zero mean over one period. It follows that

$$
Z(x, 0)=\frac{[w]}{d} x+\mathcal{C}_{d}(w-[w]) .
$$

It also follows, using the Cauchy-Riemann equations, that, when $p \geq 1$,

$$
\mathcal{G}_{d}(w)=\frac{[w]}{d}+\left(\mathcal{C}_{d}(w-[w])\right)^{\prime}=\frac{[w]}{d}+\mathcal{C}_{d}\left(w^{\prime}\right)
$$

Throughout the paper we are interested in solutions $(\Omega, \psi)$ of the water-wave problem (1.1) of class $C^{1, \alpha}$, for some $\alpha \in(0,1)$, by which which we mean that $\mathcal{S}$ has a parametrization (1.1b) with $u, v$ functions of class $C^{1, \alpha}$, such that (1.1c) holds and

$$
u^{\prime}(s)^{2}+v^{\prime}(s)^{2} \neq 0 \quad \text { for all } s \in \mathbb{R}
$$

while $\psi \in C^{\infty}(\Omega) \cap C^{1, \alpha}(\bar{\Omega})$. The main result of this section is that the free-boundary problem (1.1) is then equivalent to the problem of finding a positive number $h$ and a function $v \in C_{2 \pi}^{1, \alpha}$ which satisfy the following:

$$
\begin{aligned}
& \left\{\frac{m}{k h}+\gamma\left(\mathcal{G}_{k h}\left(v^{2} / 2\right)-v \mathcal{G}_{k h}(v)\right)\right\}^{2}=(Q-2 g v)\left(v^{\prime 2}+\mathcal{G}_{k h}(v)^{2}\right), \\
& {[v]=h,} \\
& v(x)>0 \text { for all } x \in \mathbb{R},
\end{aligned}
$$

the mapping $x \mapsto\left(\frac{x}{k}+\mathcal{C}_{k h}(v-h)(x), v(x)\right)$ is injective on $\mathbb{R}$,

$$
v^{\prime}(x)^{2}+\mathcal{G}_{k h}(v)(x)^{2} \neq 0 \text { for all } x \in \mathbb{R},
$$

where

$$
L=2 \pi / k, \quad k>0 .
$$

(Thus $2 \pi$-periodicity in $x$ becomes $L$-periodicity in $s=x / k$.) More precisely, we have the following.

Theorem 2.2. Let $(\Omega, \psi)$ be a solution of (1.1) of class $C^{1, \alpha}$. Then there exist a positive number $h$, a function $v \in C_{2 \pi}^{1, \alpha}$ and a constant $a \in \mathbb{R}$ such that (2.9) holds and

$$
\mathcal{S}=\left\{\left(a+\frac{x}{k}+\mathcal{C}_{k h}(v-h)(x), v(x)\right): x \in \mathbb{R}\right\} .
$$

Conversely, let $h>0$ and $v \in C_{2 \pi}^{1, \alpha}$ be such that (2.9) holds, and let $a \in \mathbb{R}$ be arbitrary. Let $\mathcal{S}$ be defined by (2.11), and $\Omega$ be the domain whose boundary consists of $\mathcal{S}$ and the real axis $\mathcal{B}$. Then there exists a function $\psi$ in $\Omega$ such that $(\Omega, \psi)$ is a solution of (1.1) of class $C^{1, \alpha}$.

The presence of the constant $a$ in (2.11) is due to the invariance of problem (1.1) to horizontal translations. We now explain the meaning of the positive constant $h$ which arises in (2.9). A domain $\Omega$ contained in the upper half of the $(X, Y)$-plane is called an L-periodic strip-like domain if its boundary consists of the real axis $\mathcal{B}$ and a curve $\mathcal{S}$ described in parametric form by (1.1b) such that (1.1c) holds. For any 
such domain, we define its conformal mean depth as the unique positive number $h$ such that there exists a conformal mapping $\tilde{U}+i \tilde{V}$ from the strip $\mathcal{R}_{h}$ onto $\Omega$, which admits an extension as a homeomorphism between the closures of these domains, with $\{(x, 0): x \in \mathbb{R}\}$ being mapped onto $\mathcal{S}$ and $\{(x,-h): x \in \mathbb{R}\}$ being mapped onto $\mathcal{B}$, and such that

$$
\tilde{U}(x+L, y)=\tilde{U}(x, y)+L, \quad \tilde{V}(x+L, y)=\tilde{V}(x, y), \quad(x, y) \in \mathcal{R}_{h} .
$$

The existence of a unique number $h$ with this property is proved in Appendix A. (The notion of conformal mean depth is reminiscent of that of the conformal modulus for doubly connected domains, defined as the unique number $R \in(0,1)$ such that the domain is conformally equivalent to the annulus $\left\{(x, y) \in \mathbb{R}^{2}: R^{2}<x^{2}+y^{2}<1\right\}$, see [26, 30].) Note that, for any horizontal strip, its conformal mean depth coincides with its usual depth (height). Also, as we show in Appendix A, the conformal mean depth coincides with the 'mean depth' used in [1] in the study of irrotational flows. The proof of Theorem 2.2 reveals that $h$ in (2.9) is exactly the conformal mean depth of $\Omega$.

Proof of Theorem 2.2. Let $(\Omega, \psi)$ be a solution of (1.1) of class $C^{1, \alpha}$. Let $h$ be the conformal mean depth of $\Omega$, and let $\tilde{U}+i \tilde{V}$ be the associated conformal mapping. As we show in Appendix A, it is a consequence of the Kellogg-Warschawski Theorem that $\tilde{U}, \tilde{V} \in C^{1, \alpha}\left(\overline{\mathcal{R}_{h}}\right)$ and

$$
\tilde{U}_{x}^{2}(x, 0)+\tilde{V}_{x}^{2}(x, 0) \neq 0 \quad \text { for all } x \in \mathbb{R} .
$$

Consider the mapping $U+i V: \mathcal{R}_{k h} \rightarrow \Omega$ given by

$$
U(x, y)=\tilde{U}(x / k, y / k), \quad V(x, y)=\tilde{V}(x / k, y / k), \quad(x, y) \in \mathcal{R}_{k h},
$$

where $k$ is given by (2.10). Then $U+i V$ is a conformal mapping from $\mathcal{R}_{k h}$ onto $\Omega$, which admits an extension as a homeomorphism between the closures of these domains, with $\{(x, 0): x \in \mathbb{R}\}$ being mapped onto $\mathcal{S}$ and $\{(x,-k h): x \in \mathbb{R}\}$ being mapped onto $\mathcal{B}$, and such that

$$
U(x+2 \pi, y)=U(x, y)+\frac{2 \pi}{k}, \quad V(x+2 \pi, y)=V(x, y), \quad(x, y) \in \mathcal{R}_{k h} .
$$

Moreover, $U, V \in C^{1, \alpha}\left(\overline{\mathcal{R}_{h}}\right)$ and

$$
U_{x}^{2}(x, 0)+V_{x}^{2}(x, 0) \neq 0 \quad \text { for all } x \in \mathbb{R},
$$

while the conformal mapping properties of $U+i V$ imply that

$$
V(x,-h)=0,
$$

the mapping $x \mapsto(U(x, 0), V(x, 0))$ is injective on $\mathbb{R}$,

Let

$$
\mathcal{S}=\{(U(x, 0), V(x, 0)): x \in \mathbb{R}\} .
$$

Then $v \in C_{2 \pi}^{1, \alpha}$. Since $V$ is harmonic in $\mathcal{R}_{k h}$ and satisfies (2.16) and (2.19), we deduce, by comparing (2.14) with (2.3), (2.4) and (2.5), that

$$
[v]=h .
$$

It follows, by using (2.7), that (2.17) and (2.18) can be rewritten as

$$
\begin{aligned}
& \text { the mapping } x \mapsto\left(\frac{x}{k}+\mathcal{C}_{k h}(v-h)(x), v(x)\right) \text { is injective on } \mathbb{R}, \\
& \mathcal{S}=\left\{\left(a+\frac{x}{k}+\mathcal{C}_{k h}(v-h)(x), v(x)\right): x \in \mathbb{R}\right\},
\end{aligned}
$$


for some $a \in \mathbb{R}$. Also, using the Cauchy-Riemann equations one can rewrite (2.15) as

$$
v^{\prime}(x)^{2}+\mathcal{G}_{k h}(v)(x)^{2} \neq 0 \quad \text { for all } x \in \mathbb{R} .
$$

Observe also that, since $\mathcal{S}$ is contained in the upper half-plane, it follows that

$$
v(x)>0 \text { for all } x \in \mathbb{R} .
$$

Suppose now that $\psi$ satisfies (1.1), and let $\xi: \mathcal{R}_{k h} \rightarrow \mathbb{R}$ be given by

$$
\xi(x, y)=\psi(U(x, y), V(x, y)), \quad(x, y) \in \mathcal{R}_{k h} .
$$

Note that (1.1d) can be rewritten as

$$
(X, Y) \mapsto \psi(X, Y)+\frac{\gamma}{2} Y^{2} \text { is a harmonic function in } \Omega .
$$

Hence, since harmonic functions are invariant under conformal mappings, it follows that

$$
\xi+\frac{\gamma}{2} V^{2} \text { is harmonic in } \mathcal{R}_{k h} .
$$

It is immediate from (1.1e) and (1.1f) that

$$
\begin{aligned}
& \xi(x,-k h)=-m, \quad x \in \mathbb{R}, \\
& \xi(x, 0)=0, \quad x \in \mathbb{R} .
\end{aligned}
$$

By using the chain rule and the Cauchy-Riemann equations, we easily obtain that

$$
\xi_{x}^{2}+\xi_{y}^{2}=\left(\psi_{X}^{2}(U, V)+\psi_{Y}^{2}(U, V)\right)\left(V_{x}^{2}+V_{y}^{2}\right) \quad \text { in } \overline{\mathcal{R}_{k h}} .
$$

It follows from (1.1g), taking into account (2.15), that

$$
\xi_{x}^{2}+\xi_{y}^{2}=(Q-2 g V)\left(V_{x}^{2}+V_{y}^{2}\right) \quad \text { at }(x, 0) \text { for all } x \in \mathbb{R} .
$$

Let $\zeta: \mathcal{R}_{k h} \rightarrow \mathbb{R}$ be given by

$$
\zeta=\xi+m+\frac{\gamma}{2} V^{2}
$$

Then (2.26) can be equivalently rewritten as

$$
\begin{aligned}
& \Delta \zeta=0 \quad \text { in } \mathcal{R}_{k h}, \\
& \zeta(x,-k h)=0 \text { for all } x \in \mathbb{R}, \\
& \zeta(x, 0)=m+\frac{\gamma}{2} v^{2}(x) \quad \text { for all } x \in \mathbb{R}, \\
& \left(\zeta_{y}-\gamma V V_{y}\right)^{2}=(Q-2 g V)\left(V_{x}^{2}+V_{y}^{2}\right) \text { at }(x, 0) \text { for all } x \in \mathbb{R} .
\end{aligned}
$$

Equation (2.28) can be conveniently expressed by means of the Dirichlet-Neumann operator as

$$
\left\{\frac{m}{k h}+\gamma\left(\mathcal{G}_{k h}\left(v^{2} / 2\right)-v \mathcal{G}_{k h}(v)\right)\right\}^{2}=(Q-2 g v)\left(v^{\prime 2}+\mathcal{G}_{k h}(v)^{2}\right),
$$

where we have used the linearity of $\mathcal{G}_{k h}$ and the fact that its action on constant functions is given by (2.2). By gathering (2.20), (2.21), (2.23), (2.24) and (2.29), we obtain (2.9).

Conversely, suppose that the positive number $h$ and the function $v \in C_{2 \pi}^{1, \alpha}$ satisfy (2.9). We now show how one can construct a solution of (1.1) by reversing the process which led from (1.1) to (2.9). Let $V$ be the harmonic function in $\mathcal{R}_{k h}$ which satisfies (2.16) and (2.19), and let $U: \mathcal{R}_{k h} \rightarrow \mathbb{R}$ be such that $U+i V$ is holomorphic. By Lemma 2.1, $U, V \in C^{1, \alpha}\left(\overline{\mathcal{R}_{k h}}\right)$. Condition (2.9b) ensures that (2.14) holds. Condition (2.9d) shows that the curve $\mathcal{S}$ given by (2.18), which can also be expressed as (2.22), is non-self-intersecting, and (2.9c) shows that it is contained in the upper half-plane. (The fact that $U$ is determined by $V$ only up to an additive constant leads to the presence of an arbitrary constant $a \in \mathbb{R}$ in (2.22).) Let $\Omega$ be the domain whose boundary consists of $\mathcal{S}$ and $\mathcal{B}$. An application of the Darboux-Picard Theorem 4 , 
Corollary 9.16, p. 310], see [41, Proof of Theorem 3.4] for details, shows that $U+i V$ is a conformal mapping from $\mathcal{R}_{k h}$ onto $\Omega$, which admits an extension as a homeomorphism between the closures of these domains, with $\{(x, 0): x \in \mathbb{R}\}$ being mapped onto $\mathcal{S}$ and $\{(x,-k h): x \in \mathbb{R}\}$ being mapped onto $\mathcal{B}$. It follows from (2.14) that $\Omega$ is a $L$-periodic strip-like domain, where $L$ is given by (2.10). The domain $\Omega$ has conformal mean depth $h$, since the mapping $\tilde{U}+i \tilde{V}: \mathcal{R}_{h} \rightarrow \Omega$, where $\tilde{U}, \tilde{V}$ are given by (2.13), has all the required properties. Note also that (2.9e) shows that $\mathcal{S}$ is a $C^{1, \alpha}$ curve. Let us define $\zeta$ as the unique solution of (2.28a $)-(2.28 \mathrm{c})$. Obviously, $\zeta \in C^{1, \alpha}\left(\overline{\mathcal{R}_{k h}}\right) \cap C^{\infty}\left(\mathcal{R}_{k h}\right)$. Let us define $\xi$ by (2.27), and then $\psi$ by (2.25). We obtain that $\psi \in C^{1, \alpha}(\bar{\Omega}) \cap C^{\infty}(\Omega)$ satisfies (1.1d)-(1.1f). Finally, since (2.9a) holds, we obtain that $\psi$ satisfies (1.1g). This completes the proof.

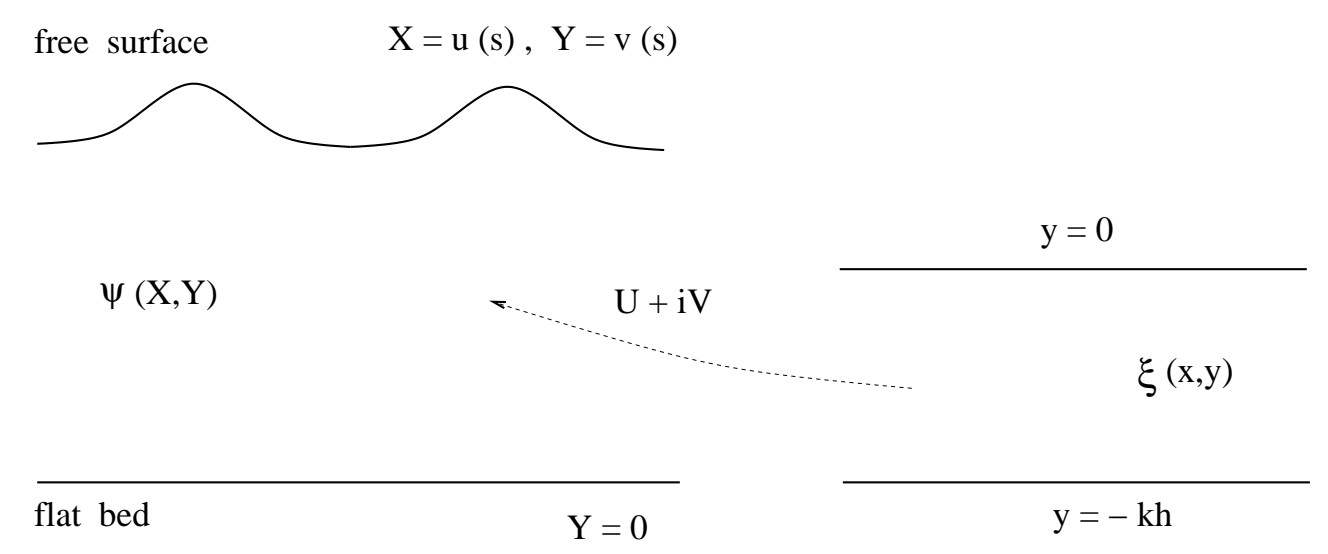

Figure 1. The conformal parametrization of the fluid domain .

For the sake of completeness, notice that differentiating (2.25), solving the resulting linear system for $\psi_{X}$ and $\psi_{Y}$ and taking subsequently (2.27) into accoount, we find that the velocity field $\left(\psi_{Y},-\psi_{X}\right)$ at the location $(X, Y)=(U(x, y), V(x, y)) \in \Omega$, where $(x, y) \in \mathcal{R}_{k h}$, can be expressed as

$$
\left(\frac{V_{x} \zeta_{x}+V_{y} \zeta_{y}}{V_{x}^{2}+V_{y}^{2}}-\gamma V, \frac{V_{x} \zeta_{y}-V_{y} \zeta_{x}}{V_{x}^{2}+V_{y}^{2}}\right)
$$

in terms of $\zeta(x, y)$ and of the conformal map $U+i V$ from $\mathcal{R}_{k h}$ to $\Omega$.

\section{ON PERIODIC HARMONIC FUNCTIONS IN A STRIP}

This section contains a more detailed investigation of the operators $\mathcal{C}_{d}$ and $\mathcal{G}_{d}$, for $d>0$, in particular their representation as singular integrals and some of its consequences. These results are important in the study, carried out in Sections 4 and 5 , of the regularity and local bifurcation of solutions of (2.9).

Let $L_{2 \pi}^{2}$ be the space of $2 \pi$-periodic locally square integrable functions of one real variable, and $W_{2 \pi}^{1,2}$ be the space of $2 \pi$-periodic locally absolutely continuous functions whose weak derivatives are in $L_{2 \pi}^{2}$. By $L_{2 \pi, \circ}^{2}$ and $W_{2 \pi, \circ}^{1,2}$ be denote the subspaces of $L_{2 \pi}^{2}$ and $W_{2 \pi}^{1,2}$ whose elements have zero mean over one period. Every function $w \in L_{2 \pi}^{2}$ has a Fourier series expansion

$$
w=[w]+\sum_{n=1}^{\infty} a_{n} \cos (n x)+\sum_{n=1}^{\infty} b_{n} \sin (n x),
$$


and, if we denote

$$
\|w\|=\left([w]^{2}+\sum_{n=1}^{\infty}\left(a_{n}^{2}+b_{n}^{2}\right)\right)^{1 / 2}
$$

then, by Parseval's Identity, $\|\cdot\|$ is an equivalent norm to the standard one in $L_{2 \pi}^{2}$.

For any $d>0$ and $w \in L_{2 \pi}^{2}$, the function $W: \mathcal{R}_{d} \rightarrow \mathbb{R}$ given by

$W(x, y)=\frac{[w]}{d}(y+d)+\sum_{n=1}^{\infty} a_{n} \frac{\sinh (n(y+d))}{\sinh (n d)} \cos (n x)+\sum_{n=1}^{\infty} b_{n} \frac{\sinh (n(y+d))}{\sinh n d} \sin (n x)$,

is the unique solution of (2.1) with the third condition there being satisfied not necessarily in the classical sense, but rather as

$$
\lim _{y \nearrow 0}\|W(\cdot, y)-w\|=0 .
$$

When $w \in W_{2 \pi}^{1,2}$, we define $\mathcal{G}_{d}(w)$ as the unique function in $L_{2 \pi}^{2}$ such that

$$
\lim _{y \nearrow 0}\left\|W_{y}(\cdot, y)-\mathcal{G}_{d}(w)\right\|=0
$$

namely

$$
\mathcal{G}_{d}(w)=\frac{[w]}{d}+\sum_{n=1}^{\infty} n a_{n} \operatorname{coth}(n d) \cos (n x)+\sum_{n=1}^{\infty} n b_{n} \operatorname{coth}(n d) \sin (n x) .
$$

The mapping $\mathcal{G}_{d}$ is the periodic Dirichlet-Neumann operator for a strip, a bounded linear operator from $W_{2 \pi}^{1,2}$ into $L_{2 \pi}^{2}$.

Suppose that $w \in L_{2 \pi, \circ}^{2}$. Then any harmonic function $Z$ in $\mathcal{R}_{d}$ such that $Z+i W$ is holomorphic is given by

$$
Z(x, y)=C+\sum_{n=1}^{\infty} a_{n} \frac{\cosh (n(y+d))}{\sinh (n d)} \sin (n x)-\sum_{n=1}^{\infty} b_{n} \frac{\cosh (n(y+d))}{\sinh n d} \cos (n x),
$$

where $C$ is a constant. We choose $C=0$ and define $\mathcal{C}_{d}(w)$ as the unique function in $L_{2 \pi, \circ}^{2}$ such that

$$
\lim _{y \nearrow 0}\left\|Z(\cdot, y)-\mathcal{C}_{d}(w)\right\|=0
$$

namely

$$
\mathcal{C}_{d}(w)=\sum_{n=1}^{\infty} a_{n} \operatorname{coth}(n d) \sin (n x)-\sum_{n=1}^{\infty} b_{n} \operatorname{coth}(n d) \cos (n x) .
$$

The mapping $\mathcal{C}_{d}$ is the periodic Hilbert transform for a strip, a bounded linear operator from $L_{2 \pi, \circ}^{2}$ into itself. Moreover, one can easily see that $\mathcal{C}_{d}$ is a bijection from $L_{2 \pi, \circ}^{2}$ onto itself. Let us denote by $\mathcal{C}_{d}^{-1}$ its inverse. Observe that, by formally setting $d=\infty$ in (3.6), we obtain the familiar periodic Hilbert transform [7, 31, 38,

$$
\mathcal{C}(w)=\sum_{n=1}^{\infty} a_{n} \sin (n x)-\sum_{n=1}^{\infty} b_{n} \cos (n x)
$$

for all $w \in L_{2 \pi, \circ}^{2}$ with the Fourier expansion (3.1). The operator $\mathcal{C}$ is a bijection from $L_{2 \pi, \circ}^{2}$ onto itself, and $\mathcal{C}^{-1}=-\mathcal{C}$.

The operator $\mathcal{C}$ has a pointwise almost everywhere representation as a singular integral

$$
\mathcal{C}(w)(t)=\frac{1}{2 \pi} P V \int_{-\pi}^{\pi} \cot \left(\frac{t-s}{2}\right) w(s) d s,
$$

where $P V$ denotes a principal value integral 32. The representation (3.8) is useful in proving Privalov's Theorem [24]: for every $\alpha \in(0,1)$ the operator $\mathcal{C}$ is a bounded 
linear operator from $C_{2 \pi}^{0, \alpha}$ into itself. Moreover, (3.8) is also instrumental in proving (see [7, Lemma 10.5.3, p. 148]) that the operator

$$
w \mapsto \mathcal{Q}(w)=w \mathcal{C}\left(w^{\prime}\right)-\mathcal{C}\left(w w^{\prime}\right)
$$

maps functions $w \in C_{2 \pi}^{1, \alpha}$ into functions in $C_{2 \pi}^{1, \delta}$ for any $\delta \in(0, \alpha)$. We now prove that these results, and the corresponding ones for functions of higher regularity, extend to the case of $\mathcal{C}_{d}$, for any $d>0$, by exhibiting an analogous representation of $\mathcal{C}_{d}$ as a singular integral. Let us denote by $\mathcal{Q}_{d}$ the mapping

$$
w \mapsto \mathcal{Q}_{d}(w)=w \mathcal{C}_{d}\left(w^{\prime}\right)-\mathcal{C}_{d}\left(w w^{\prime}\right)
$$

Lemma 3.1. For any $d>0, p \geq 0$ integer and $\alpha \in(0,1), \mathcal{C}_{d}$ is a bounded linear operator from $C_{2 \pi, \mathrm{o}}^{p, \alpha}$ into itself. Moreover, $\mathcal{C}_{d}^{-1}$ is also a bounded linear operator from $C_{2 \pi, o}^{p, \alpha}$ into itself.

Lemma 3.2. If $w \in C_{2 \pi}^{p, \alpha}$ with $p \geq 1$ integer and $\alpha \in(0,1)$, then $\mathcal{Q}_{d}(w) \in C_{2 \pi}^{p, \delta}$ for any $\delta \in(0, \alpha)$.

Observe that the operators $\mathcal{C}$ and $\mathcal{C}_{d}$ can be extended by (complex-)linearity to complex-valued functions in $L_{2 \pi, 0}^{2}$, and are characterized by their action on the trigonometric system $\left\{e^{i n t}\right\}_{n \in \mathbb{Z} \backslash\{0\}}$ :

$$
\mathcal{C}\left(e^{i n t}\right)=-i \operatorname{sgn}(n) e^{i n t}, \quad n \in \mathbb{Z} \backslash\{0\},
$$

and

$$
\mathcal{C}_{d}\left(e^{i n t}\right)=-i \operatorname{coth}(n d) e^{i n t}, \quad n \in \mathbb{Z} \backslash\{0\} .
$$

Let us write

$$
\mathcal{C}_{d}=\mathcal{C}+\mathcal{K}_{d}
$$

Then the operator $\mathcal{K}_{d}$ corresponds to the Fourier multiplier operator on $L_{2 \pi, 0}^{2}$ :

$$
\left\{w=\sum_{n \in \mathbb{Z} \backslash\{0\}} c_{n} e^{i n t}\right\} \mapsto\left\{\sum_{n \in \mathbb{Z} \backslash\{0\}}-i \operatorname{sgn}(n) \lambda_{n} c_{n} e^{i n t}\right\}
$$

with

$$
\lambda_{n}=\frac{2}{e^{2|n| d}-1}, \quad|n| \geq 1 .
$$

Notice that for any $n \geq 1$,

$$
e^{2 n d}-1=\sum_{m=0}^{\infty} \frac{1}{m !} n^{m}(2 d)^{m} \geq \frac{1}{(p+1) !} n^{p+1}(2 d)^{p+1}, \quad p \geq 1,
$$

yields

$$
0 \leq \frac{n^{p}}{e^{2 n d}-1} \leq \frac{1}{n} \frac{(p+1) !}{(2 d)^{p+1}}, \quad p \geq 1,
$$

so that

$$
\sum_{n \in \mathbb{Z} \backslash\{0\}}|n|^{2 p} \lambda_{n}^{2} \leq 4\left(\frac{(p+1) !}{(2 d)^{p+1}}\right)^{2} \sum_{n \in \mathbb{Z} \backslash\{0\}} \frac{1}{n^{2}}<\infty .
$$

Let $\kappa_{d} \in L_{2 \pi, \circ}^{2}$ be given by

$$
\kappa_{d}(t)=\sum_{n \in \mathbb{Z} \backslash\{0\}}-i \operatorname{sgn}(n) \lambda_{n} e^{i n t}=\sum_{n=1}^{\infty} 2 \lambda_{n} \sin (n t), \quad t \in \mathbb{R} .
$$


Since the Fourier coefficients of $\kappa_{d}$ decays faster than any power of $|n|, \kappa_{d}$ is a function of class $C^{\infty}$ (see [17]). It follows from (3.14) that $\mathcal{K}_{d}(w)$ is the periodic convolution of $w$ with the smooth function $\kappa_{d}$ given by (3.15), namely

$$
\mathcal{K}_{d}(w)(t)=\frac{1}{2 \pi} \int_{-\pi}^{\pi} \kappa_{d}(t-s) w(s) d s, \quad t \in \mathbb{R} .
$$

Proof of Lemma 3.1. It is easy to see that $\mathcal{K}_{d}$ is a bounded linear operator from $C_{2 \pi, \circ}^{p, \alpha}$ into itself. The operator $\mathcal{C}$ is also a bounded linear operator from $C_{2 \pi, 0}^{p, \alpha}$ into itself, by Privalov's Theorem and the fact that $\mathcal{C}$ commutes with differentiation on $C_{2 \pi, 0}^{1, \alpha}$. The required result for $\mathcal{C}_{d}$ follows from (3.13). The corresponding result for $\mathcal{C}_{d}^{-1}$ is obtained by the same argument, after observing that one can write

$$
\mathcal{C}_{d}^{-1}=-\mathcal{C}+\tilde{\mathcal{K}}_{d}
$$

where $\tilde{\mathcal{K}}_{d}$ is given by periodic convolution with a smooth function.

Proof of Lemma 3.2. Using (3.10) and (3.16), we write

$$
\mathcal{Q}_{d}(w)=\mathcal{Q}(w)+\left\{w \mathcal{K}_{d}\left(w^{\prime}\right)-\mathcal{K}_{d}\left(w w^{\prime}\right)\right\} .
$$

Since $\kappa_{d}$ is smooth, it is easy to see from (3.16) that $\mathcal{K}_{d}\left(w^{\prime}\right)$ and $\mathcal{K}_{d}\left(w w^{\prime}\right)$ are both smooth functions. The required result follows then from (3.18), provided that one can show that $\mathcal{Q}$ maps $C_{2 \pi}^{p, \delta}$ into $C_{2 \pi}^{p, \delta}$ for any $\delta \in(0, \alpha)$. This fact has been proved in 7] for $p=1$, and in [28] for $p \geq 2$, the proof in [28] being in fact in a more general setting.

For the sake of completeness, we now give a self-contained proof of the fact that, for any $p \geq 1$ integer, $\mathcal{Q}$ maps $C_{2 \pi}^{p, \delta}$ into $C_{2 \pi}^{p, \delta}$ for any $\delta \in(0, \alpha)$. Our proof relies on a commutator estimate proved in Appendix B, which is more general and has a more transparent proof than the results in [28]. Let $w \in C_{2 \pi}^{p, \delta}$, with $p \geq 1$ integer and $\alpha \in(0,1)$. Since $w^{(p)} \in C_{2 \pi}^{0, \alpha}$, we may interchange the operators $\mathcal{C}$ and differentiation to infer from Leibniz's rule that

$$
\left(w \mathcal{C}\left(w^{(p)}\right)-\mathcal{C}\left(w w^{(p)}\right)\right)-\partial_{x}^{p-1}\left(w \mathcal{C}\left(w^{\prime}\right)-\mathcal{C}\left(w w^{\prime}\right)\right) \in C_{2 \pi}^{1, \alpha} .
$$

Thus it suffices to show that for any $\delta \in(0, \alpha)$ we have

$$
\left(w \mathcal{C}\left(w^{(p)}\right)-\mathcal{C}\left(w w^{(p)}\right)\right) \in C_{2 \pi}^{1, \delta}
$$

However, estimate (3.19) is an immediate consequence of Lemma B.1 in Appendix B. This completes the proof.

Remark 3.3. Since for $\alpha \in(0,1)$ the function

$$
w:[-\pi, \pi] \rightarrow \mathbb{R}, \quad w(x)=\frac{x}{\alpha+1}\left(|x|^{\alpha}-\pi^{\alpha}\right),
$$

extends by periodicity to a function $w \in C_{2 \pi}^{1, \alpha}$ with $w \mathcal{C}\left(w^{\prime}\right)-\mathcal{C}\left(w w^{\prime}\right) \notin C_{2 \pi}^{1, \alpha}$ (see the detailed estimates in [28]), in view of (3.16) we see that the result of Lemma 3.2 cannot be improved to accommodate $\delta=\alpha$.

\section{Regularity}

The main result of this section concerns regularity of solutions of (2.9).

Theorem 4.1. Let $h>0$ and $v \in C_{2 \pi}^{1, \alpha}$ be a solution of (2.9) such that

$$
Q-2 g v(x)>0 \quad \text { for all } x \in \mathbb{R} \text {. }
$$

Then $v \in C_{2 \pi}^{\infty}$. 
Remark 4.2. Theorem 4.1 implies that, is $(\Omega, \psi)$ is a solution of (1.1) of class $C^{1, \alpha}$ with no stagnation points on the free surface, then $\mathcal{S}$ is a $C^{\infty}$ curve and $\psi \in C^{\infty}(\bar{\Omega})$. Indeed, by Theorem 2.2, any such solution gives rise to a solution of (2.9), the absence of stagnation points on $\mathcal{S}$ being equivalent to (4.1). By Theorem 4.1, $v \in C_{2 \pi}^{\infty}$ and, by Lemma 3.1, $\mathcal{C}_{k h}(v-h) \in C_{2 \pi}^{\infty}$. Hence $\mathcal{S}$, being given by (2.11), is a $C^{\infty}$ curve, and the fact that $\psi \in C^{\infty}(\bar{\Omega})$ follows from standard elliptic regularity theory [18].

Remark 4.3. Our proof of Theorem 4.1 is based on harmonic analysis estimates and is relatively simple. Using different methods which are somewhat more involved, the stronger conclusion that $s \mapsto v(s)$ is real-analytic on $\mathbb{R}$ can be obtained. Indeed, once the $C^{2, \alpha}$ regularity of $\mathcal{S}$ and of $\psi$ in $\bar{\Omega}$ is known (for example from Theorem 4.1), the approach of Kinderlehrer, Nirenberg and Spruck [21] is applicable, as first observed by Constantin and Escher [11], showing that the curve $\mathcal{S}$ is real-analytic. Then a standard result of regularity of conformal mappings up to the boundary [29] shows that $U+i V$ has a holomorphic extension to a neighbourhood of the real axis, implying in particular that $s \mapsto v(s)$ is real-analytic on $\mathbb{R}$. An alternative proof of this fact, based on ordinary differential equations in the complex domain in the spirit of Lewy's theorem [25], has been pointed out to us by the anonymous referee of this paper.

Proof of Theorem 4.1. Let $U, V$ be as in the proof of the second part of Theorem 2.2. with $U, V \in C^{1, \alpha}\left(\overline{\mathcal{R}_{k h}}\right)$. As noted there, $U+i V$ is a conformal mapping from $\mathcal{R}_{k h}$ onto $\Omega$, and therefore

$$
U_{x}+i V_{x} \neq 0 \quad \text { in } \mathcal{R}_{k h} .
$$

Since $V>0$ in $\mathcal{R}_{k h}$ and $V(x,-k h)=0$ for all $x \in \mathbb{R}$, it follows from Hopf boundarypoint lemma [18] that

$$
V_{y}(x,-k h)>0 \quad \text { for all } x \in \mathbb{R} .
$$

Taking also into account (2.9e), we have thus obtained that

$$
U_{x}+i V_{x} \neq 0 \quad \text { in } \overline{\mathcal{R}_{k h}} \text {. }
$$

One can therefore write

$$
U_{x}+i V_{x}=\left(U_{x}^{2}+V_{x}^{2}\right)^{1 / 2} e^{i \theta},
$$

where $\theta \in C^{0, \alpha}\left(\overline{\mathcal{R}_{h}}\right)$ is such that

$$
\begin{gathered}
\log \left(U_{x}^{2}+V_{x}^{2}\right)^{1 / 2}+i \theta \quad \text { is holomorphic in } \mathcal{R}_{k h}, \\
\theta(x,-k h)=0 \quad \text { for all } x \in \mathbb{R} .
\end{gathered}
$$

Let

$$
\theta_{0}(x)=\theta(x, 0), \quad u(x)=U(x, 0), \quad x \in \mathbb{R} .
$$

Then

$$
\mathcal{C}_{k h}\left(\theta_{0}\right)=\log \left(u^{\prime 2}+v^{\prime 2}\right)^{1 / 2}-\left[\log \left(u^{\prime 2}+v^{\prime 2}\right)^{1 / 2}\right]
$$

so that

$$
\theta_{0}=\mathcal{C}_{k h}^{-1}\left(\log \left(u^{\prime 2}+v^{\prime 2}\right)^{1 / 2}-\left[\log \left(u^{\prime 2}+v^{\prime 2}\right)^{1 / 2}\right]\right) .
$$

Since (4.1) holds, equation (2.9a) can be written as

$$
\left(u^{\prime 2}+v^{\prime 2}\right)^{1 / 2}=\frac{\left|\frac{m}{k h}+\gamma\left\{\mathcal{G}_{k h}\left(v^{2} / 2\right)-v \mathcal{G}_{k h}(v)\right\}\right|}{(Q-2 g v)^{1 / 2}} .
$$

Notice that in view of (2.8), (2.9b) and (3.10), we have

$$
\mathcal{G}_{k h}\left(v^{2} / 2\right)-v \mathcal{G}_{k h}(v)=\frac{\left[v^{2}\right]}{2 k h}+\mathcal{C}_{k h}\left(v v^{\prime}\right)-\frac{v}{k}-v \mathcal{C}_{k h}\left(v^{\prime}\right)=\frac{\left[v^{2}\right]}{2 k h}-\frac{v}{k}-\mathcal{Q}_{k h}(v) .
$$


Since $v \in C_{2 \pi}^{1, \alpha}$, Lemma 3.2 ensures that $\mathcal{Q}_{k h}(v) \in C_{2 \pi}^{1, \alpha / 2}$ and from (4.6) and (4.7) we infer that $\left(u^{\prime 2}+v^{\prime 2}\right) \in C_{2 \pi}^{1, \alpha / 2}$. Using this in (4.5), Lemma 3.1 ensures now that $\theta_{0} \in C_{2 \pi}^{1, \alpha / 2}$. But

$$
\left\{\begin{array}{l}
u^{\prime}=\left(u^{\prime 2}+v^{\prime 2}\right)^{1 / 2} \cos \theta_{0}, \\
v^{\prime}=\left(u^{\prime 2}+v^{\prime 2}\right)^{1 / 2} \sin \theta_{0},
\end{array}\right.
$$

so that $u, v \in C_{2 \pi}^{2, \alpha / 2}$. Implementing this procedure $p$ times we obtain that

$$
u, v \in C_{2 \pi}^{1+p, \alpha / 2^{p}}, \quad p \geq 1 .
$$

Consequently $v \in C_{2 \pi}^{\infty}$. This completes the proof.

\section{LOCAL BIFURCATION}

In this section we prove the existence of solutions of (2.9). Because of (2.9b), it is natural to put

$$
v=w+h,
$$

and rewrite (2.9), taking into account (2.8), as

$$
\begin{aligned}
& \left\{\frac{m}{k h}+\gamma\left(\frac{\left[w^{2}\right]}{2 k h}-\frac{w}{k}-\frac{h}{2 k}+\mathcal{C}_{k h}\left(w w^{\prime}\right)-w \mathcal{C}_{k h}\left(w^{\prime}\right)\right)\right\}^{2}= \\
& \quad=(Q-2 g h-2 g w)\left\{w^{\prime 2}+\left(\frac{1}{k}+\mathcal{C}_{k h}\left(w^{\prime}\right)\right)^{2}\right\}, \\
& {[w]=0,} \\
& w(x)>-h \text { for all } x \in \mathbb{R},
\end{aligned}
$$

the mapping $x \mapsto\left(\frac{x}{k}+\mathcal{C}_{k h}(w)(x), w(x)+h\right)$ is injective on $\mathbb{R}$,

$$
w^{\prime}(x)^{2}+\left(\frac{1}{k}+\mathcal{C}_{k h}\left(w^{\prime}\right)(x)\right)^{2} \neq 0 \quad \text { for all } x \in \mathbb{R},
$$

We prove, for each $\gamma \in \mathbb{R}, k>0$ and $h>0$ fixed, and regarding $m$ and $Q$ as parameters, the existence of solutions $w \in C_{2 \pi}^{1, \alpha}$ of (5.2). Note that, if

$$
\frac{1}{k}+\mathcal{C}_{k h}\left(w^{\prime}\right)(x)>0 \quad \text { for all } x \in \mathbb{R},
$$

then (5.2d) and (5.2e) are automatically satisfied and, moreover, the corresponding free boundary $\mathcal{S}$ is the graph of a function. Motivated by the remark that $w$ sufficiently small in $C_{2 \pi}^{1, \alpha}$ ensures the validity of (5.2c) and (5.3), we concentrate on proving the existence of small-amplitude solutions $w \in C_{2 \pi, \circ}^{1, \alpha}$ of (5.2a). Of course, any study of large-amplitude solutions of (5.2a) $-(5.2 \mathrm{~b})$ will need to discuss also the validity of (5.2c)-(5.2e), which is necessary for the construction of solutions of (1.1).

Note that $w=0 \in C_{2 \pi, o}^{1, \alpha}$ is a solution of (5.2a) if and only if

$$
Q=2 g h+\left(\frac{m}{h}-\frac{\gamma h}{2}\right)^{2} .
$$

This suggests setting

$$
\begin{aligned}
& \lambda=\frac{m}{h}-\frac{\gamma h}{2}, \\
& \mu=Q-2 g h-\left(\frac{m}{h}-\frac{\gamma h}{2}\right)^{2} .
\end{aligned}
$$


The mapping $(m, Q) \mapsto(\lambda, \mu)$ is a bijection from $\mathbb{R}^{2}$ onto itself. Equations (5.2a)(5.2b) can be rewritten as

$$
\begin{aligned}
& \left\{\frac{\lambda}{k}+\gamma\left(\frac{\left[w^{2}\right]}{2 k h}-\frac{w}{k}+\mathcal{C}_{k h}\left(w w^{\prime}\right)-w \mathcal{C}_{k h}\left(w^{\prime}\right)\right)\right\}^{2}= \\
& =\left(\lambda^{2}+\mu-2 g w\right)\left\{w^{\prime 2}+\left(\frac{1}{k}+\mathcal{C}_{k h}\left(w^{\prime}\right)\right)^{2}\right\},
\end{aligned}
$$

with $w \in C_{2 \pi, o}^{1, \alpha}, \mu \in \mathbb{R}$ and $\lambda \in \mathbb{R}$. Note that $w=0 \in C_{2 \pi, \circ}^{1, \alpha}$ and $\mu=0$ is a solution of (5.6) for every $\lambda \in \mathbb{R}$.

To prove the existence of solutions of (5.6) we apply the Crandall-Rabinowitz theorem [15] on bifurcation from a simple eigenvalue. For a linear operator $\mathcal{L}$ between two Banach spaces, let us denote by $\mathcal{N}(\mathcal{L})$ its null space and by $\mathcal{R}(\mathcal{L})$ its range.

Theorem 5.1 (Local bifurcation theorem [15]). Let $\mathbb{X}$ and $\mathbb{Y}$ be Banach spaces, $I$ an open interval in $\mathbb{R}$ containing $\lambda^{*}$, and $F \in C(I \times \mathbb{X}, \mathbb{Y})$. Suppose that

(i) $F(\lambda, 0)=0$ for all $\lambda \in I$;

(ii) $\partial_{\lambda} F, \partial_{u} F$, and $\partial_{\lambda, u}^{2} F$ exist and are continuous;

(iii) $\mathcal{N}\left(\partial_{u} F\left(\lambda^{*}, 0\right)\right)$ and $\mathbb{Y} / \mathcal{R}\left(\partial_{u} F\left(\lambda^{*}, 0\right)\right)$ are one-dimensional, with the nullspace generated by $u^{*}$;

(iv) the transversality condition $\partial_{\lambda, u}^{2} F\left(\lambda^{*}, 0\right)\left(1, u^{*}\right) \notin \mathcal{R}\left(\partial_{u} F\left(\lambda^{*}, 0\right)\right)$ holds.

Then there exists a continuous local bifurcation curve $\{(\lambda(s), u(s)):|s|<\varepsilon\}$ with $\varepsilon>0$ sufficiently small such that $(\lambda(0), u(0))=\left(\lambda^{*}, 0\right)$ and

$$
\{(\lambda, u) \in \mathcal{O}: u \neq 0, F(\lambda, u)=0\}=\{(\lambda(s), u(s)): 0<|s|<\varepsilon\}
$$

for some neighborhood $\mathcal{O}$ of $\left(\lambda^{*}, 0\right) \in I \times \mathbb{X}$. Moreover, we have

$$
u(s)=s u^{*}+o(s) \quad \text { in } \quad \mathbb{X},|s|<\varepsilon,
$$

and if $\partial_{u}^{2} F$ is also continuous, then the curve is of class $C^{1}$, while for $F$ of class $C^{k}$ $(k \geq 2)$ or real-analytic, $s \mapsto u(s)$ is of class $C^{k-1}$, respectively real-analytic.

Let us denote, for every integer $p \geq 0$,

$$
\begin{gathered}
C_{2 \pi, e}^{p, \alpha}=\left\{f \in C_{2 \pi}^{p, \alpha}: f(x)=f(-x) \text { for all } x \in \mathbb{R}\right\}, \\
C_{2 \pi, \circ, e}^{p, \alpha}=\left\{f \in C_{2 \pi, \circ}^{p, \alpha}: f(x)=f(-x) \text { for all } x \in \mathbb{R}\right\} .
\end{gathered}
$$

To apply the local bifurcation theorem to (5.6), let

$$
\mathbb{X}=\mathbb{R} \times C_{2 \pi, o, e}^{p+1, \alpha}, \quad \mathbb{Y}=C_{2 \pi, e}^{p, \alpha},
$$

for some $p \geq 0$. Then we can write (5.6) as $F(\lambda,(\mu, w))=0$ with $F: \mathbb{R} \times \mathbb{X} \rightarrow \mathbb{Y}$ real-analytic given by

$$
\begin{aligned}
F(\lambda,(\mu, w))= & \gamma^{2}\left(\mathcal{C}_{k h}\left(w w^{\prime}\right)-w \mathcal{C}_{k h}\left(w^{\prime}\right)-\frac{w}{k}+\frac{\left[w^{2}\right]}{2 k h}\right)^{2} \\
& +\frac{2 \lambda \gamma}{k}\left(\mathcal{C}_{k h}\left(w w^{\prime}\right)-w \mathcal{C}_{k h}\left(w^{\prime}\right)-\frac{w}{k}+\frac{\left[w^{2}\right]}{2 k h}\right) \\
& +(2 g w-\mu)\left(\frac{1}{k^{2}}+w^{\prime 2}+\frac{2}{k} \mathcal{C}_{k h}\left(w^{\prime}\right)+\left(\mathcal{C}_{k h}\left(w^{\prime}\right)\right)^{2}\right) \\
& -\lambda^{2}\left(w^{\prime 2}+\frac{2}{k} \mathcal{C}_{k h}\left(w^{\prime}\right)+\left(\mathcal{C}_{k h}\left(w^{\prime}\right)\right)^{2}\right)
\end{aligned}
$$

As noted earlier, $F(\lambda,(0,0))=0$ for all $\lambda \in \mathbb{R}$. Taking into account Lemma 3.1, we easily compute

$$
\partial_{(\mu, w)} F(\lambda,(0,0))(f, \nu)=\frac{2}{k^{2}}\left((g-\lambda \gamma) f-\lambda^{2} k \mathcal{C}_{k h}\left(f^{\prime}\right)\right)-\frac{\nu}{k^{2}}, \quad(f, \nu) \in \mathbb{X} .
$$


It follows from the representation (3.6) and Lemma 3.1 that the bounded linear operator $\partial_{(\mu, w)} F(\lambda,(0,0)): \mathbb{X} \rightarrow \mathbb{Y}$ is invertible whenever $\lambda$ does not satisfy

$$
\lambda^{2} n k \operatorname{coth}(n k h)=g-\lambda \gamma,
$$

for any integer $n \geq 1$. Hence all potential bifurcation points for (5.6) are to be found among the solutions of (5.12) for some integer $n \geq 1$.

Suppose now that $\lambda^{*}$ is a solution of (5.12) for some integer $n \geq 1$. (Observe that (5.12) has exactly two different solutions for each $n \geq 1$.) Using again the representation (3.6) and Lemma 3.1, it follows that $\mathcal{N}\left(\partial_{(\mu, w)} F\left(\lambda^{*},(0,0)\right)\right)$ is onedimensional and generated by $\left(0, w^{*}\right) \in \mathbb{X}$, where $w^{*}(x)=\cos (n x)$ for all $x \in \mathbb{R}$, while $\mathcal{R}\left(\partial_{(\mu, w)} F\left(\lambda^{*},(0,0)\right)\right)$ is the closed subspace of $\mathbb{Y}$ formed by the functions $f \in \mathbb{Y}$ satisfying

$$
\int_{-\pi}^{\pi} f(x) \cos (n x) d x=0
$$

so that $\mathbb{Y} / \mathcal{R}\left(\partial_{(\mu, w)} F\left(\lambda^{*},(0,0)\right)\right)$ is the one-dimensional subspace of $\mathbb{Y}$ generated by the function $w^{*}(x)=\cos (n x)$. Using (5.11), we now compute

$\partial_{\lambda,(\mu, w)}^{2} F\left(\lambda^{*},(0,0)\right)\left(1,\left(0, w^{*}\right)\right)=\frac{2}{k^{2}}\left(-\gamma-2 \lambda^{*} n k \operatorname{coth}(n k h)\right) w^{*} \notin \mathcal{R}\left(\partial_{(\mu, w)} F\left(\lambda^{*},(0,0)\right)\right)$

since, using (5.12), we have

$$
-\gamma-2 \lambda^{*} n k \operatorname{coth}(n k h)=-\lambda^{*}\left(n k \operatorname{coth}(n k h)+\frac{g}{\left(\lambda^{*}\right)^{2}}\right) \neq 0 .
$$

Therefore, by the local bifurcation theorem, the solutions of (5.12) for any integer $n \geq 1$ are bifurcation points. Motivated by the quest for solutions of (5.6) of minimal period $2 \pi$, we take $n=1$ in (5.12), obtaining the bifurcation values

$$
\lambda_{ \pm}=-\frac{\gamma \tanh (k h)}{2 k} \pm \sqrt{\frac{\gamma^{2} \tanh ^{2}(k h)}{4 k^{2}}+g \frac{\tanh (k h)}{k}}
$$

The corresponding values of $m$ are obtained from (5.5a),

$$
m_{ \pm}=\frac{\gamma h^{2}}{2}-\frac{\gamma h \tanh (k h)}{2 k} \pm h \sqrt{\frac{\gamma^{2} \tanh ^{2}(k h)}{4 k^{2}}+g \frac{\tanh (k h)}{k}}
$$

The existence of water waves of small amplitude is now immediate.

Theorem 5.2. Given $h>0, k>0, \gamma \in \mathbb{R}$ and $m \in \mathbb{R}$ there exist laminar flow 1 with a flat free surface in water of depth $h$, of constant vorticity $\gamma$ and relative mass flux $m$. Moreover, the values $m_{ \pm}$of the flux given by (5.14) trigger the appearance of periodic steady waves of small amplitude, with period $2 \pi / k$ and conformal mean depth $h$, which have a smooth profile with one crest and one trough per period, monotone between consecutive crests and troughs and symmetric about any crest line. The laminar flows of flux $m_{ \pm}$are exactly those with horizontal speeds at the flat free surface $\lambda_{ \pm}$given by (5.13).

Proof of Theorem 5.2. The function $w=0$ satisfies (5.2) for any value of $m \in \mathbb{R}$, provided that $Q$ is given by (5.4). These solutions correspond to laminar flows in the fluid domain bounded below by the rigid bed $\mathcal{B}$ and above by the free surface $Y=h$, with stream function

$$
\psi(X, Y)=-\frac{\gamma}{2} Y^{2}+\left(\frac{m}{h}+\frac{\gamma h}{2}\right) Y-m, \quad X \in \mathbb{R}, 0 \leq Y \leq h,
$$

and velocity field

$$
\left(\psi_{Y},-\psi_{X}\right)=\left(-\gamma Y+\frac{m}{h}+\frac{\gamma h}{2}, 0\right), \quad X \in \mathbb{R}, 0 \leq Y \leq h .
$$

\footnotetext{
${ }^{1}$ In the sense that the water flows in layers parallel to the flat bed (with zero vertical fluid velocity).
} 
Observe that for these flows the horizontal velocity at the free surface is indeed $\lambda$ given by (5.5a).

Consider now the local bifurcation curve

$$
\{(\lambda(s),(0+o(s), s \cos (x)+o(s))):|s|<\varepsilon\} \subset \mathbb{R} \times \mathbb{X}
$$

of solutions of (5.6), issuing from the points $\left(\lambda_{ \pm},(0,0)\right)$, with $\lambda_{ \pm}$given by (5.13). If $\varepsilon>0$ is chosen small enough, then conditions (5.2C) and (5.3) are satisfied, where we have used Lemma 3.1. Therefore the corresponding non-flat free surface $\mathcal{S}$ given by (2.11) with

$$
v=w+h,
$$

is the graph of a smooth function, symmetric with respect to the points corresponding to $x=n \pi, n \in \mathbb{Z}$. If we choose $p$ in (5.9) such that $p \geq 1$, then, since

$$
w(x ; s)=s \cos (x)+o(s) \quad \text { in } C_{2 \pi}^{p+1, \alpha},
$$

we can ensure that

$$
s w^{\prime}(x ; s)<0 \text { for all } x \in(0, \pi), 0<|s|<\varepsilon,
$$

by choosing $\varepsilon>0$ suitably small. Together with the evenness of $x \mapsto w(x ; s)$, this proves the $\mathcal{S}$ has one crest and one trough per minimal period and is monotone between consecutive crests and troughs.

Let us consider the family of laminar flows from which bifurcation of small-amplitude periodic waves was shown to occur. It follows from (5.15) that they have the form

$$
\left(\psi_{Y},-\psi_{X}\right)=\left(\lambda_{ \pm}+\gamma(h-Y), 0\right), \quad X \in \mathbb{R}, 0 \leq Y \leq h .
$$

where $\lambda_{ \pm}$is given by (5.13). The formula (5.13), giving the speed $\lambda_{ \pm}$at the free surface in terms of the depth $h$, period $2 \pi / k$ and vorticity $\gamma$, is called the dispersion relation. As far as we are aware, its first occurrences in the literature are in [35] and [3], where it was obtained by formal arguments.

A remarkable feature of some of these flows is that they contain stagnation points. Since the flows are laminar, stagnation points, if present, form horizontal lines. An elementary analysis, similar to that in [44], shows that small-amplitude waves bifurcating from these flows have a critical layer. The streamline pattern is considerably different in the case when stagnation points are present compared to the case when they are not. A typical case of a flow without stagnation points is depicted in Figure 2 (see the discussion in [12]).

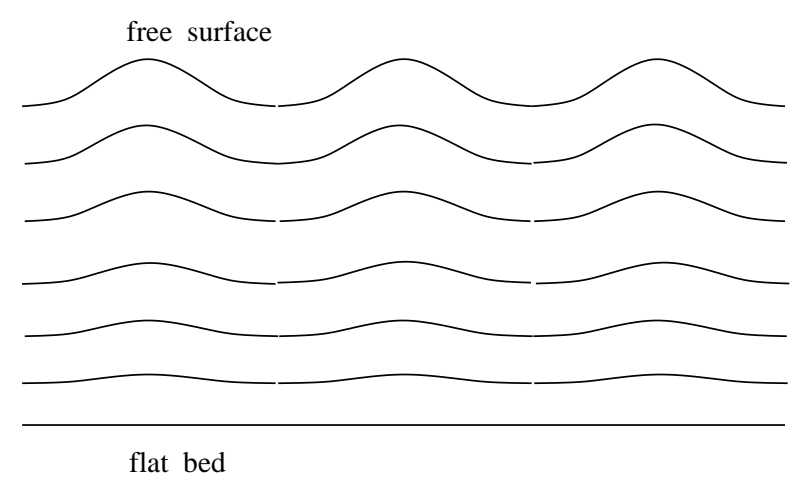

Figure 2. Flow pattern (streamlines) without stagnation points . 
A picture of the streamline pattern if the flow presents stagnation points is provided in Figure 3: the 'cat's eye' flow pattern of Thomson (Lord Kelvin) 34] is highly typical for this situation (see the discussion in [44]).

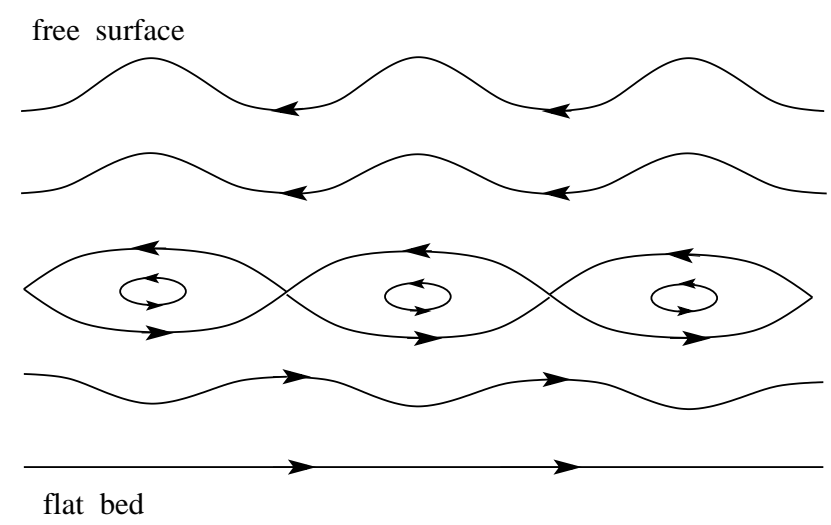

Figure 3. Flow pattern (streamlines) with stagnation points :

Kelvin's 'cat's eye' flow pattern

Notice that $\lambda_{+}>0$ and $\lambda_{-}<0$ irrespective of the values of $h, k$ and $\gamma$, so stagnation points can never occur at the free surface of the bifurcation-inducing laminar flows. This implies that in the irrotational case $(\gamma=0)$ stagnation points do not occur anywhere in these flows. In the general case stagnation points occur if and only if the equation

$$
\lambda_{ \pm}+\gamma(h-Y)=0
$$

has a solution $Y$ in $[0, h]$. It is easy to see that this is the case if and only if

$$
\lambda_{ \pm}\left(\lambda_{ \pm}+\gamma h\right) \leq 0 .
$$

Suppose first that $\gamma>0$. Then $\lambda_{+}+\gamma h>0$, so the flow corresponding to $\lambda_{+}$never contains stagnation points. The flow corresponding to $\lambda_{-}$contains stagnation points if and only if $\lambda_{-}+\gamma h \geq 0$, which is equivalent to

$$
\frac{\tanh (k h)}{k h} \leq \frac{\gamma^{2} h}{g+\gamma^{2} h} .
$$

For fixed $h$, the left-hand side of (5.19) is monotone as a function of $k$, with limit 1 as $k \rightarrow 0$ and limit 0 as $k \rightarrow \infty$, while the right-hand side is monotone as a function of $\gamma$, with limit 0 as $\gamma \rightarrow 0$ and limit 1 as $\gamma \rightarrow \infty$. Hence, for fixed $h$ and $\gamma$, there exists a unique $k^{*}>0$ for which equality holds in (5.19), which corresponds to a laminar flow stagnant at the bottom, and for $k<k^{*}$ the flow does not have stagnation points, while for $k>k^{*}$ the flow has a line of stagnation points. On the other hand, if $h$ and $k$ are fixed, then there exists a unique $\gamma^{*}>0$ for which equality holds in (5.19), which corresponds to a laminar flow stagnant at the bottom, and for $\gamma<\gamma^{*}$ the flow does not have stagnation points, while for $\gamma>\gamma^{*}$ the flow has a line of stagnation points. Whenever (5.19) holds, the stagnation line is $Y=Y_{0}$, where

$$
h-Y_{0}=\frac{\tanh (k h)}{2 k}+\sqrt{\frac{\tanh ^{2}(k h)}{4 k^{2}}+\frac{g}{\gamma^{2}} \frac{\tanh (k h)}{k}}
$$

from which we see that the distance between the stagnation line and the free surface decreases with $k$ and with $\gamma^{2}$, and approaches 0 if and only if $k \rightarrow \infty$ and $\gamma^{2} \rightarrow \infty$.

Suppose now that $\gamma<0$. This case is the mirror image of the previous one. The flow corresponding to $\lambda_{-}$never contains stagnation points. The flow corresponding to 
$\lambda_{+}$contains stagnation points if and only if (5.19) holds, in which case the stagnation line is $Y=Y_{0}$, where $Y_{0}$ satisfies (5.20).

It is of interest to compare Theorem 5.2 with the local bifurcation results for waves of constant vorticity $\gamma$ that were obtained in [12] and in [44. Let us fix $k>0$. Varying $h>0$ in Theorem 5.2 we obtain all periodic traveling wave solutions of small amplitude and minimal period $2 \pi / k$ that lie in a neighborhood of a laminar flow: for each $h$ we obtain in the space $(h, m,(\mu, w)) \in(0, \infty) \times \mathbb{R} \times \mathbb{X}$ two smooth curves through the points $\left(h, m_{-}(h), 0\right)$ and $\left(h, m_{+}(h), 0\right)$ with $m_{\mp}$ given by (5.14). The union of these curves gives a surface $\mathcal{M} \subset(0, \infty) \times \mathbb{R} \times \mathbb{X}$.

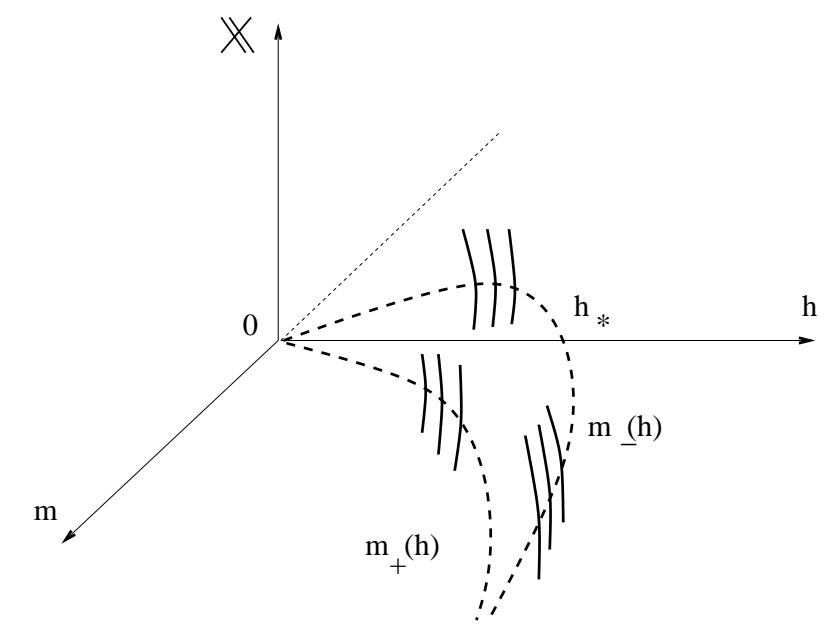

Figure 4 . The case $\gamma>0$ : The dashed curves represent the laminar bifurcating flows

Each nontrivial bifurcation curve (in bold) lies in a hyperplane $\mathrm{h}=$ constant .

In Figure 4 we depict the situation in the case $\gamma>0$ :

(i) In the $(h, m)$-plane the curve $h \mapsto m_{+}(h)$ starts at the origin and approaches the parabola $m=\frac{\gamma h^{2}}{2}$ as $h \rightarrow \infty$, while $m_{+}(h)>0$ for $h>0$. As pointed out in the lines preceding (5.19), the nontrivial flows that bifurcate from the laminar flows corresponding to $m_{+}(h)$ do not present stagnation points.

(ii) In the $(h, m)$-plane the curve $h \mapsto m_{-}(h)$ also starts at the origin and approaches the parabola $m=\frac{\gamma h^{2}}{2}$ as $h \rightarrow \infty$. Denoting by $h_{*}>0$ the unique positive solution of the equation

$$
\frac{\tanh (k h)}{k h}=\frac{\gamma^{2} h}{4 g+2 \gamma^{2} h}
$$

we see from (5.14) that $m_{-}(h)<0$ for $h \in\left(0, h_{*}\right)$ and $m_{-}(h)>0$ for $h>h_{*}$. Moreover, since

$$
\frac{\gamma^{2} h_{*}}{4 g+2 \gamma^{2} h_{*}}<\frac{\gamma^{2} h_{*}}{g+\gamma^{2} h_{*}}
$$

and the function $h \mapsto \frac{\tanh (k h)}{k h}$ is strictly decreasing on $(0, \infty)$ while $h \mapsto \frac{\gamma^{2} h}{g+\gamma^{2} h}$ is strictly increasing, we deduce that the nontrivial flows bifurcating from the laminar flows at $m_{-}(h)$ contain stagnation points if and only if $h>h^{*}$, where $h^{*} \in\left(0, h_{*}\right)$ is the unique solution to the equation obtained by requiring equality in (5.19).

The approach in 44] is very different from ours, in that the author performs a flattening $(X, Y) \mapsto\left(X, \frac{Y}{\eta(X)}\right)$ of the free surface $Y=\eta(X)$. (This method is 
therefore restricted to waves with non-overhanging profiles.) The bifurcation-inducing laminar flows identified there are exactly the same as ours. However, from the family of laminar flows of any depth $h_{0}$, the local bifurcation curve constructed in [44] issuing from $\left(h_{0}, m_{ \pm}\left(h_{0}\right)\right)$ is a curve on $\mathcal{M}$ for which

$$
Q=2 g h_{0}+\left(\frac{m}{h_{0}}-\frac{\gamma h_{0}}{2}\right)^{2}
$$

for the corresponding solutions of (1.1), but no information on the conformal mean depth of the fluid domains is obtained (so that it need not be $h_{0}$ ). There is a remark in 44 that a modification of the method there could have led to the existence of a new curve on $\mathcal{M}$, for which, instead of (5.21), the condition

$$
h_{0}=\frac{k}{2 \pi} \int_{-\pi / k}^{\pi / k} \eta(X) d X
$$

will hold for the corresponding solutions of (1.1).

The approach in 12, while more general in that it covers also non-constant vorticities, requires that there are no stagnation points in the flow. More precisely, it relies on a hodograph transform, for the existence of which it is essential that the horizontal velocity never vanishes in the flow. In [12] the relative mass flux $m$ is fixed and $h$ varies, so each nontrivial bifurcation curve on $\mathcal{M}$ is contained in a hyperplane $m=$ constant. Since 12 only studies solutions for which $\psi_{Y}<0$ in $\bar{\Omega}$, it only obtains $\lambda_{-}$in (5.14) as a bifurcation point, and only in those cases for which the associated laminar flow (5.15) does not have stagnation points. (However, the same method is easily seen to be applicable also for solutions for which $\psi_{Y}>0$ in $\bar{\Omega}$, leading to $\lambda_{+}$in (5.14) as a bifurcation point, though again only in those cases for which the associated laminar flow (5.15) does not have stagnation points.) In 12, Section 3] it was concluded that, for $k=1$, local bifurcation from trivial flows always occurs for negative constant vorticity, while in the case of positive constant vorticity there is a necessary and sufficient condition on the value of the mass flux $m<0$, namely

$$
\tanh \left(\sqrt{\frac{-2 m}{\gamma}}\right)>\frac{-2 m \gamma}{g+\gamma \sqrt{-2 m \gamma}}
$$

Let us check these results against ours. We are thus looking at the laminar bifurcationinducing flows for $k=1$, and with $\psi_{Y}<0$ everywhere in the closure of the fluid domain. Hence, by (5.13), (5.14) and (5.18), and dropping the subscripts,

$$
\begin{gathered}
\lambda=-\frac{\gamma \tanh (h)}{2}-\sqrt{\frac{\gamma^{2} \tanh ^{2}(h)}{4}+g \tanh (h),} \\
m=\frac{\gamma h^{2}}{2}-\frac{\gamma h \tanh (h)}{2}-h \sqrt{\frac{\gamma^{2} \tanh ^{2}(h)}{4}+g \tanh (h),} \\
\lambda+\gamma h<0 .
\end{gathered}
$$

We are interested in the range of the mapping $h \mapsto m(h)$ given by (5.25), restricted to the set of $h \in(0, \infty)$ for which (5.26) holds. We will prove that the range is $(-\infty, 0)$ if $\gamma<0$ and coincides with the set for which (5.23) holds, thus confirming the conclusions of [12. The case $\gamma<0$ is easy, so we concentrate on the case $\gamma>0$. Recall that (5.24) was obtained from (5.12) with $n=1$ and $k=1$, hence

$$
\lambda^{2}=(g-\gamma \lambda) \tanh (h)
$$

as the solution with

$$
\lambda<0,
$$


while (5.25) was obtained from (5.24) by, see (5.5a),

$$
m=\lambda h+\frac{\gamma h^{2}}{2} \text {. }
$$

Hence $m$ is in the required range if and only if (5.27) and (5.29) are solvable with respect to $\lambda \in \mathbb{R}$ and $h>0$ so that (5.26) and (5.28) are satisfied. We solve first (5.29) with respect to $h$ for fixed $\lambda$ satisfying (5.28). The condition

$$
\lambda \leq-\sqrt{-2 \gamma m}
$$

is necessary and sufficient for solvability, and the requirement (5.26) leads to the unique solution

$$
h=-\frac{\lambda}{\gamma}-\frac{\sqrt{\lambda^{2}+2 \gamma m}}{\gamma},
$$

and to strict inequality in (5.30). Plugging (5.31) into (5.27) we obtain

$$
\tanh \left(-\frac{\lambda}{\gamma}-\frac{\sqrt{\lambda^{2}+2 \gamma m}}{\gamma}\right)=\frac{\lambda^{2}}{g-\gamma \lambda},
$$

to be solved with respect to $\lambda$ in the interval $(-\infty,-\sqrt{-2 \gamma m})$. The necessary and sufficient condition for this solvability is easily seen to be exactly (5.23), as required.

\section{APpendix A. On CONFORMAL MAPPINGS FOR $L$-PERIODIC STRIP-LIKE DOMAINS}

We prove here the existence and uniqueness of the conformal mean depth for any $L$ periodic strip-like domain $\Omega$. For any such domain $\Omega$, let $\Omega^{R}$ be the domain obtained from $\Omega$ by symmetrization with respect to the real axis $\mathcal{B}$, and let

$$
\tilde{\Omega}=\Omega \cup \mathcal{B} \cup \Omega^{R} \text {. }
$$

Let $H>0$ be such that $\tilde{\Omega}$ is a subset of the horizontal strip

$$
S_{H}=\left\{(x, y) \in \mathbb{R}^{2}:-H<y<H\right\} .
$$

The application

$$
\Phi(z)=\frac{e^{\frac{\pi}{2 H} z}-1}{e^{\frac{\pi}{2 H}} z+1}
$$

maps $S_{H}$ conformally onto the unit disc $\mathbb{D}=\{\xi \in \mathbb{C}:|\xi|<1\}$. Let $\Pi=\Phi(\tilde{\Omega})$. The boundary of $\Pi$ is a Jordan curve $\mathcal{J} \subset \mathbb{D} \cup\{ \pm 1\}$, symmetric about the real axis and passing through the points \pm 1 . Clearly $\Phi$ is a homeomorphism between the closures of $\tilde{\Omega}$ and of $\Pi$. Moreover, if $\mathbb{D}_{+}$denotes the part of $\mathbb{D}$ situated in the upper half-plane and $\Pi_{+}=\Pi \cap \mathbb{D}_{+}$, then $\Pi_{+}=\Phi(\Omega)$. Carathéodory's theorem [29, Theorem 2.6 and Corollary 2.6, p. 24] ensures the existence and uniqueness of a conformal map $\Phi_{1}$ from $\mathbb{D}$ onto $\Pi$, which has an extension as a homeomorphism between the closures of these domains and is such that $\Phi_{1}( \pm 1)= \pm 1$ and $\Phi_{1}(0)=0$. The uniqueness assertion implies that $\Phi_{1}(\bar{z})=\overline{\Phi_{1}(z)}$ for all $z \in \mathbb{D}$. This in turn implies that $\Phi_{1}$ maps the segment $[-1,1] \subset \mathbb{R}$ onto itself, and the imaginary part of $\Phi_{1}$ does not change sign in $\mathbb{D}_{+}$. Since the real part of $\Phi_{1}$ is strictly increasing on the segment $[-1,1] \subset \mathbb{R}$, the Cauchy-Riemann equations on that segment imply that the imaginary part of $\Phi_{1}$ is positive in $\mathbb{D}_{+}$. Hence $\Phi_{1}$ maps $\mathbb{D}_{+}$onto $\Pi_{+}$. Denote $\Phi_{0}=\Phi^{-1}$. Since

$$
\Phi_{2}(z)=\frac{e^{\frac{\pi}{2}(z+i)}-1}{e^{\frac{\pi}{2}(z+i)}+1}
$$

maps $\mathcal{R}_{2}$ conformally onto $\mathbb{D}$, we see that $\Phi_{0} \circ \Phi_{1} \circ \Phi_{2}=U_{0}+i V_{0}$ with $U_{0}, V_{0}: \mathcal{R}_{2} \rightarrow \mathbb{R}$, is a conformal mapping of $\mathcal{R}_{2}$ onto $\tilde{\Omega}$, with an extension as a homeomorphism between the closures of these domains, such that $\mathcal{R}_{1}$ is mapped onto $\Omega$ and points symmetric 
in $\mathcal{R}_{2}$ with respect to the line $y=-1$ are mapped into points in $\tilde{\Omega}$ that are symmetric with respect to the real axis.

Let $\beta+i \delta$ be the inverse of $U_{0}+i V_{0}$, with $\beta, \delta: \tilde{\Omega} \rightarrow \mathbb{R}$. Since $\Omega$ is an $L$-periodic strip-like domain, we observe that the function

$$
(X, Y) \mapsto \delta(X+L, Y)-\delta(X, Y)
$$

is harmonic in $\Omega$, and has a continuous extension to the closure of $\Omega$ which is zero on the boundary of $\Omega$ since by construction $\delta=-1$ on $\mathcal{B}$ and $\delta=0$ on $\mathcal{S}$. Being also bounded in $\Omega$ (since by construction $|\delta| \leq 1$ ), the Phragmen-Lindelöf principle [17] ensures that this function is identically zero, so that

$$
\delta(X+L, Y)=\delta(X, Y), \quad(X, Y) \in \Omega .
$$

The Cauchy-Riemann equations then imply that

$$
\beta(X+L, Y)-\beta(X, Y)=K, \quad(X, Y) \in \Omega,
$$

for some constant $K \in \mathbb{R}$. Note that necessarily $K>0$, since the fact that the real part of $\Phi_{1}$ is a strictly increasing homeomorphism of the segment $[-1,1] \subset \mathbb{D}$ implies that $x \mapsto \beta(x, 0)$ is a strictly increasing homeomorphism of $\mathbb{R}$. From (A.1) and (A.2) we infer that

$$
\left\{\begin{array}{l}
U_{0}(x+K, y)=U_{0}(x, y)+L, \\
V_{0}(x+K, y)=V_{0}(x, y),
\end{array} \quad(x, y) \in \mathcal{R}_{1} .\right.
$$

Replacing $U_{0}+i V_{0}$ from $\mathcal{R}_{1}$ onto $\Omega$ by $\tilde{U}+i \tilde{V}: \mathcal{R}_{h} \rightarrow \Omega$ given by

$$
\tilde{U}(x, y)=U_{0}(x / h, y / h), \quad \tilde{V}(x, y)=V_{0}(x / h, y / h), \quad(x, y) \in \mathcal{R}_{h},
$$

where

$$
h=L / K,
$$

we see that $h$ satisfies the definition of the conformal mean depth of $\Omega$.

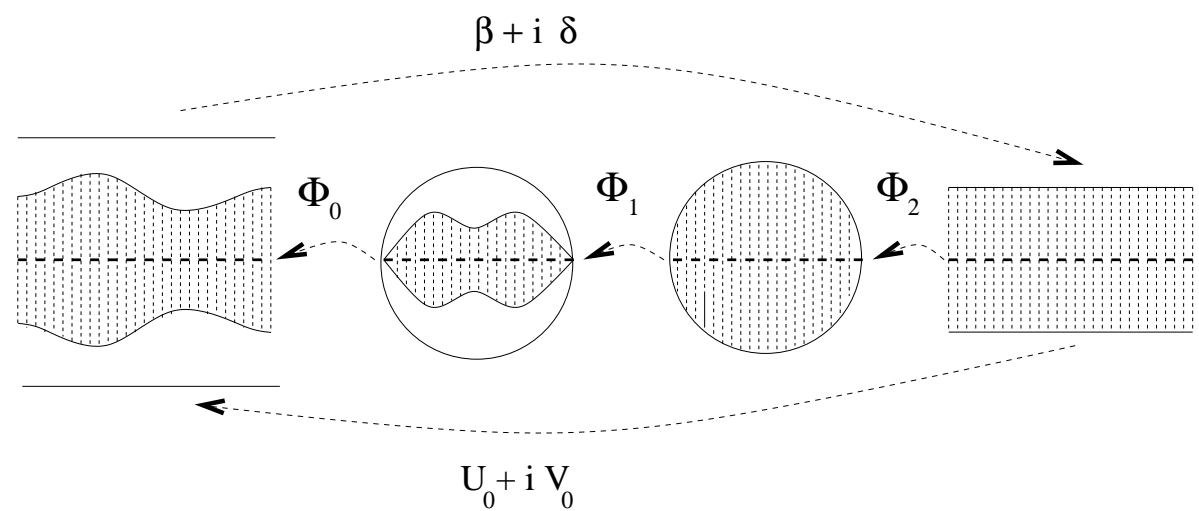

Successive steps in the construction of the conformal map.

Suppose now that $\hat{h}$ also satisfies this definition, and let $\hat{U}+i \hat{V}: \mathcal{R}_{\hat{h}} \rightarrow \Omega$ be the corresponding conformal mapping. Then

$$
(\hat{U}+i \hat{V})^{-1} \circ(\tilde{U}+i \tilde{V})
$$

is a conformal bijection from $\mathcal{R}_{h}$ onto $\mathcal{R}_{\hat{h}}$ and a homeomorphism between the closures of these domains. Let us write

$$
(\hat{U}+i \hat{V})^{-1} \circ(\tilde{U}+i \tilde{V})=\tau+i \omega,
$$


where $\tau, \omega: \mathcal{R}_{h} \rightarrow \mathbb{R}$ are harmonic functions. It follows that

$$
\left\{\begin{array}{l}
\tau(x+L, y)=\tau(x, y)+L, \\
\omega(x+L, y)=\omega(x, y),
\end{array} \quad(x, y) \in \mathcal{R}_{h} .\right.
$$

Also,

$$
\omega(x, 0)=0, \quad \omega(x,-h)=-\hat{h}, \quad x \in \mathbb{R} .
$$

The maximum principle shows that

$$
\omega(x, y)=\hat{h} y / h, \quad(x, y) \in \mathcal{R}_{h},
$$

which implies that

$$
\tau(x, y)=(\hat{h} x / h)+C, \quad(x, y) \in \mathcal{R}_{h},
$$

where $C \in \mathbb{R}$ is a constant, a fact which, together with the first relation in (A.4) implies that $\hat{h}=h$.

If, in addition, $\mathcal{S}$ is a curve of class $C^{1, \alpha}$, then the Jordan curve $\mathcal{J}$ is $C_{\text {loc }}^{1, \alpha}$ everywhere with exception of the two points \pm 1 . By the Kellogg-Warschawski theorem [29, Theorems 3.5 and 3.6, p. 48], the conformal map $\Phi_{1}$ inherits this smoothness up to the boundary, namely it is of class $C_{\text {loc }}^{1, \alpha}$ in the closure of $\mathbb{D}$ with the exception of \pm 1 and, moreover, its derivative is not zero at any point in $\overline{\mathbb{D}} \backslash\{ \pm 1\}$. Our construction ensures, taking into account the periodicity, that $\tilde{U}, \tilde{V} \in C^{1, \alpha}\left(\overline{\mathcal{R}_{h}}\right)$ and

$$
\tilde{U}_{x}^{2}+\tilde{V}_{x}^{2} \neq 0 \quad \text { in } \overline{\mathcal{R}_{h}} .
$$

We now show that $h$ coincides with the 'mean depth' of a $L$-periodic strip-like domain as defined in [1]. There, the domain $\Omega$ is considered by as being occupied by a fluid in steady irrotational motion, so that $\mathcal{S}$ and $\mathcal{B}$ are streamlines for a stream function $\psi$ which is $L$-periodic in $X$ throughout $\Omega$ and satisfies (1.1d) with $\gamma=0$, (1.1e) and (1.1f). Since the flow is irrotational, there exists an associated velocity potential $\phi$ in $\Omega$, which is a harmonic function such that $\phi+i \psi$ is holomorphic. The velocity field is then given by $\left(\psi_{Y},-\psi_{X}\right)$, which also equals $\left(\phi_{X}, \phi_{Y}\right)$ by the Cauchy-Riemann equations. The mean horizontal velocity of the flow is then given by

$$
c=\frac{1}{L} \int_{X}^{X+L} \phi_{X}(T, Y) d T=\frac{1}{L}(\phi(X+L, Y)-\phi(X, Y)),
$$

a quantity which is easily seen not to depend on $(X, Y)$ as long as the horizontal line segment joining $(X, Y)$ and $(X+L, Y)$ is contained in $\Omega$. The 'mean depth' is then defined as $m / c$, the ratio between the flux and the mean horizontal velocity. With $\beta, \delta$ as previously defined in Appendix A, note that the maximum principle implies that

$$
\psi(X, Y)=m \delta(X, Y), \quad(X, Y) \in \Omega,
$$

and then the Cauchy-Riemann equations show that

$$
\phi(X+L, Y)-\phi(X, Y)=m(\beta(X+L, Y)-\beta(X, Y)), \quad(X, Y) \in \Omega .
$$

Therefore

$$
c L=m K
$$

and hence

$$
h=m / c .
$$

Consequently the conformal mean depth of $\Omega$ coincides with the 'mean depth' defined in [1]. 
Appendix B. A commutator estimate

Lemma B.1. If $f \in C_{2 \pi}^{1, \alpha}$ and $g \in C_{2 \pi}^{0, \alpha}$ with $\alpha \in(0,1)$, then

$$
f \mathcal{C}(g)-\mathcal{C}(f g) \in C_{2 \pi}^{1, \delta} \quad \text { for all } \delta \in(0, \alpha) .
$$

Proof. Let

$$
\Theta(t)=f(t) \mathcal{C}(g)(t)-\mathcal{C}(f g)(t) \quad \text { for all } t \in \mathbb{R}
$$

Using the definition of $\mathcal{C}$ we obtain that, for all $t \in \mathbb{R}$,

$$
\Theta(t)=\frac{1}{2 \pi} \int_{-\pi}^{\pi} \cot \left(\frac{t-s}{2}\right)\{f(t)-f(s)\} g(s) d s,
$$

where, since $f \in C_{2 \pi}^{1, \alpha}$, the integral is not a principal value integral, but a genuine Lebesgue integral. In proving that $\Theta \in C_{2 \pi}^{1, \delta}$ for all $\delta \in(0, \alpha)$ we will make use of the following inequalities:

$$
\begin{gathered}
|x-\sin (x)| \leq \frac{1}{6}|x|^{3} \quad \text { for all } x \in \mathbb{R}, \\
\frac{1}{\sin ^{2}(x)} \leq \sigma(|x|) \frac{1}{x^{2}} \quad \text { for all } x \text { with } 0<|x|<\pi, \\
\left|\cot (x)-\frac{1}{x}\right| \leq \varsigma(|x|)|x| \quad \text { for all } x \text { with } 0<|x|<\pi,
\end{gathered}
$$

where $\sigma, \varsigma:(0, \pi) \rightarrow[0, \infty)$ are bounded functions on $(0, a]$ for each $a \in(0, \pi)$.

We show first that $\Theta$ is differentiable on $\mathbb{R}$ and its derivative is given, for all $t \in \mathbb{R}$, by the formula obtained by formal differentiation under the integral sign:

$$
\Theta^{\prime}(t)=\frac{1}{4 \pi} \int_{-\pi}^{\pi} \frac{f^{\prime}(t) \sin (t-s)-\{f(t)-f(s)\}}{\sin ^{2}\left(\frac{t-s}{2}\right)} g(s) d s .
$$

Note that the above integral finite. Indeed, the integral

$$
\frac{1}{4 \pi} \int_{t-\pi}^{t+\pi} \frac{f^{\prime}(t) \sin (t-s)-\{f(t)-f(s)\}}{\sin ^{2}\left(\frac{t-s}{2}\right)} g(s) d s
$$

is finite, as it can be seen upon using $(\overline{B .2}),(\overline{B .3})$ and the Mean Value Theorem, by which

$$
\begin{aligned}
\left|f^{\prime}(t)(t-s)-\{f(t)-f(s)\}\right| & =|t-s|\left|f^{\prime}(t)-f^{\prime}(\xi)\right| \\
& \leq C|t-s|^{1+\alpha},
\end{aligned}
$$

for some $\xi=\xi(t, s)$ with $|\xi-t| \leq|s-t|$. Hence the integral in (B.5) is finite, being obtained from that in (B.6) by a rearrangement using the periodicity of the functions involved. In the above and throughout what follows, we denote by $C$ a constant which depends only on the norm of $f$ in $C_{2 \pi}^{1, \alpha}$ and the norm of $g$ in $C_{2 \pi}^{0, \alpha}$, and is allowed to vary from line to line.

Since the operator defining $\Theta$ commutes with translations, it suffices to show that $\Theta$ is differentiable at $t=0$, with derivative at $t=0$ given by (B.5). Note that, for any $t$ close to 0 , one can write

$$
2 \pi \frac{\Theta(t)-\Theta(0)}{t}=I_{1}(t)+I_{2}(t)
$$

where

$$
\begin{gathered}
I_{1}(t)=\frac{1}{t} \int_{-2|t|}^{2|t|}\left\{\cot \left(\frac{t-s}{2}\right)\{f(t)-f(s)\}-\cot \left(\frac{-s}{2}\right)\{f(0)-f(s)\}\right\} g(s) d s \\
I_{2}(t)=\int_{(-\pi,-2|t|) \cup(2|t|, \pi)} \frac{1}{t}\left\{\cot \left(\frac{t-s}{2}\right)\{f(t)-f(s)\}-\cot \left(\frac{-s}{2}\right)\{f(0)-f(s)\}\right\} g(s) d s .
\end{gathered}
$$


Using (B.4), one can write $I_{1}(t)$ as the sum of a term which tends to 0 as $t \rightarrow 0$ and

$$
\frac{2}{t} \int_{-2|t|}^{2|t|}\left\{\frac{f(t)-f(s)}{t-s}-\frac{f(0)-f(s)}{0-s}\right\} g(s) d s
$$

Since, for fixed $t$, the integrand in the above is continuous as a function of $s$, the Mean Value Theorem for integrals shows that the above quantity equals

$$
\frac{8|t|}{t}\left\{\frac{f(t)-f(\xi)}{t-\xi}-\frac{f(0)-f(\xi)}{0-\xi}\right\} g(\xi)
$$

for some $\xi \in(-2|t|, 2|t|)$. Using again the Mean Value Theorem, the above quantity equals

$$
\frac{8|t|}{t}\left\{f^{\prime}\left(\xi_{1}\right)-f^{\prime}\left(\xi_{2}\right)\right\} g(\xi)
$$

for some $\xi_{1}, \xi_{2} \in(-2|t|, 2|t|)$. Since $f^{\prime}$ is continuous and $g$ is bounded, the above quantity tends to 0 as $t \rightarrow 0$. We have thus proved that

$$
I_{1}(t) \rightarrow 0 \quad \text { as } t \rightarrow 0 .
$$

We now prove that, as $t \rightarrow 0$,

$$
I_{2}(t) \rightarrow \int_{-\pi}^{\pi} \frac{f^{\prime}(0) \sin (-s)-\{f(0)-f(s)\}}{2 \sin ^{2}\left(\frac{s}{2}\right)} g(s) d s .
$$

For this, we use the Dominated Convergence Theorem. Observe that $I_{2}(t)$ can be written as an integral over $(-\pi, \pi)$, with the integrand given by

$$
\chi_{(-\pi,-2|t|) \cup(2|t|, \pi)}\left(\frac{f(t)-f(0)}{t} \cot \left(\frac{t-s}{2}\right)+\{f(0)-f(s)\} \frac{\cot \left(\frac{t-s}{2}\right)-\cot \left(-\frac{s}{2}\right)}{t}\right) g(s)
$$

where $\chi_{A}$ stands for the characteristic function of the set $A$. Firstly, note that, as $t \rightarrow 0$, the above integrand converges pointwise to the integrand on the right-hand side of (B.8). Secondly, note that, for each $s \in(-\pi, 0) \cup(0, \pi)$ and for each $t$ with $0<|t|<|s| / 2$, the Mean Value Theorem yields that

$$
\begin{gathered}
\frac{1}{t}\left\{\cot \left(\frac{t-s}{2}\right)\{f(t)-f(s)\}-\cot \left(\frac{-s}{2}\right)\{f(0)-f(s)\}\right\} \\
=\frac{f^{\prime}(\xi) \sin (\xi-s)-\{f(\xi)-f(s)\}}{2 \sin ^{2}\left(\frac{\xi-s}{2}\right)}
\end{gathered}
$$

for some $\xi$ between 0 and $t$. Using again $(\underline{B .2})$ and (B.3), we obtain

$$
\begin{aligned}
\left|\frac{f^{\prime}(\xi) \sin (\xi-s)-\{f(\xi)-f(s)\}}{2 \sin ^{2}\left(\frac{\xi-s}{2}\right)}\right| & \leq C\left|\frac{f^{\prime}(\xi)(\xi-s)-\{f(\xi)-f(s)\}}{(\xi-s)^{2}}\right|+C|\xi-s| \\
& =C\left|\frac{f^{\prime}(\xi)-f^{\prime}\left(\xi_{0}\right)}{\xi-s}\right|+C|\xi-s| \\
& \leq C|\xi-s|^{\alpha-1} \\
& \leq C|s|^{\alpha-1},
\end{aligned}
$$

where $\xi$ is between 0 and $t, \xi_{0}$ is between $\xi$ and $s$, and we took into account that $0<|t|<|s| / 2$ and $f \in C_{2 \pi}^{1, \alpha}$. Since $s \mapsto|s|^{\alpha-1}$ is integrable and $g$ is bounded, we have thus checked the conditions of the Dominated Convergence Theorem. Therefore, (B.8) holds and, since (B.7) holds, it follows that $\Theta$ is differentiable at $t=0$, with derivative given by (B.5) evaluated at $t=0$. As noted earlier, this implies that $\Theta$ is differentiable on $\mathbb{R}$, with derivative given by (B.5). 
We now prove that $\Theta^{\prime} \in C_{2 \pi}^{0, \delta}$ for each $\delta \in(0, \alpha)$. Since $\Theta^{\prime}$ commutes with translation, it suffices to show that

$$
\left|\Theta^{\prime}(t)-\Theta^{\prime}(0)\right| \leq C|t|^{\delta} \quad \text { for all } t \text { close to } 0 .
$$

The change of variables $s \mapsto(t-s)$ in (B.5) leads to the following formula

$$
\Theta^{\prime}(t)=\frac{1}{4 \pi} \int_{-\pi}^{\pi} \frac{f^{\prime}(t-s) \sin (s)-\{f(t)-f(t-s)\}}{\sin ^{2}\left(\frac{s}{2}\right)} g(t-s) d s,
$$

for all $t \in \mathbb{R}$. It follows that, for each $t$ close to 0 , one can write

$$
4 \pi\left(\Theta^{\prime}(t)-\Theta^{\prime}(0)\right)=J_{1}(t)+J_{2}(t)+J_{3}(t),
$$

where

$$
\begin{gathered}
J_{1}(t)=\int_{-2|t|}^{2|t|} \frac{f^{\prime}(t) \sin (s)-\{f(t)-f(t-s)\}}{\sin ^{2}\left(\frac{s}{2}\right)} g(t-s) d s \\
J_{2}(t)=-\int_{-2|t|}^{2|t|} \frac{f^{\prime}(0) \sin (s)-\{f(0)-f(-s)\}}{\sin ^{2}\left(\frac{s}{2}\right)} g(-s) d s \\
J_{3}(t)=\int_{(-\pi,-2|t|) \cup(2|t|, \pi)}\left\{\frac{f^{\prime}(t) \sin (s)-\{f(t)-f(t-s)\}}{\sin ^{2}\left(\frac{s}{2}\right)} g(t-s)\right. \\
\left.-\frac{f^{\prime}(0) \sin (s)-\{f(0)-f(-s)\}}{\sin ^{2}\left(\frac{s}{2}\right)} g(-s)\right\} d s .
\end{gathered}
$$

Note first that

$$
\begin{aligned}
\left|J_{1}(t)\right| & \leq \int_{-2|t|}^{2|t|}\left|f^{\prime}(t)\right||g(t-s)| \frac{|\sin (s)-s|}{\sin ^{2}\left(\frac{s}{2}\right)}+\int_{-2|t|}^{2|t|}|g(t-s)| \frac{\left|\int_{-s}^{0}\left\{f^{\prime}(t)-f^{\prime}(t+\tau)\right\} d \tau\right|}{\sin ^{2}\left(\frac{s}{2}\right)} d s \\
& \leq C|t|^{2}+C|t|^{\alpha} \\
& \leq C|t|^{\alpha}
\end{aligned}
$$

where we have used $(\overline{B .3})$ and $(\overline{B .2})$, the boundedness of $f^{\prime}$ and $g$, and the fact that $f^{\prime} \in C_{2 \pi}^{0, \alpha}$. Then, exactly the same argument gives that

$$
\left|J_{2}(t)\right| \leq C|t|^{\alpha} .
$$

Also, one can write

$$
J_{3}(t)=K_{1}(t)+K_{2}(t),
$$

where

$$
\begin{aligned}
& K_{1}(t)=\int_{(-\pi,-2|t|) \cup(2|t|, \pi)} \frac{\left\{f^{\prime}(t)-f^{\prime}(0)\right\} \sin (s)+\{f(0)-f(-s)\}-\{f(t)-f(t-s)\}}{\sin ^{2}\left(\frac{s}{2}\right)} g(t-s) d s \\
& K_{2}(t)=\int_{(-\pi,-2|t|) \cup(2|t|, \pi)} \frac{f^{\prime}(0) \sin (s)-\{f(0)-f(-s)\}}{\sin ^{2}\left(\frac{s}{2}\right)}\{g(t-s)-g(-s)\} d s .
\end{aligned}
$$

Using (B.3), the boundedness of $g$ and the fact that $f^{\prime} \in C_{2 \pi}^{0, \alpha}$, it follows that

$$
\begin{aligned}
\left|K_{1}(t)\right| & \leq C \int_{(-\pi,-2|t|) \cup(2|t|, \pi)}|g(t-s)|\left\{\frac{\left|f^{\prime}(t)-f^{\prime}(0)\right|}{|s|}+\frac{\left|\int_{-s}^{0}\left(f^{\prime}(\tau)-f^{\prime}(t+\tau)\right) d \tau\right|}{|s|^{2}}\right\} d s \\
& \leq C|t|^{\alpha} \int_{(-\pi,-2|t|) \cup(2|t|, \pi)} \frac{1}{|s|} d s \\
& \leq C|t|^{\alpha}|\log | t|| \\
& \leq C|t|^{\delta} .
\end{aligned}
$$


Also, using (B.3), the boundedness of $f^{\prime}$ and the the fact that $g \in C_{2 \pi}^{0, \alpha}$, it follows that

$$
\begin{aligned}
\left|K_{2}(t)\right| & \leq C \int_{(-\pi,-2|t|) \cup(2|t|, \pi)}|g(t-s)-g(-s)|\left\{\frac{\left|f^{\prime}(0)\right|}{|s|}+\frac{\left|\int_{-s}^{0} f^{\prime}(\tau) d \tau\right|}{|s|^{2}}\right\} d s \\
& \leq C|t|^{\alpha} \int_{(-\pi,-2|t|) \cup(2|t|, \pi)} \frac{1}{|s|} d s \\
& \leq C|t|^{\alpha}|\log | t|| \\
& \leq C|t|^{\delta}
\end{aligned}
$$

Combining the estimates (B.12)-(B.17) yields (B.10). As noted earlier, this implies that $\Theta^{\prime} \in C_{2 \pi}^{0, \delta}$. This completes the proof.

Acknowledgement. The support of the Vienna Science and Technology Fund (WWTF) is gratefully acknowledged. The authors thank the referee for a useful suggestion concerning the regularity result in Section 4.

\section{REFERENCES}

[1] C. J. Amick and J. F. Toland, On periodic water-waves and their convergence to solitary waves in the long-wave limit, Phil. Trans. Roy. Soc. London Ser. A 303 (1981), 633-669.

[2] K. I. Babenko, Some remarks on the theory of surface waves of finite amplitude, Dokl. Akad. Nauk 294 (1987), 1033-1037.

[3] F. Biesel, Etude théorique de la houle en eau courante, Houille Blanche, 5 (1950), 279-285.

[4] R. B. Burckel, An introduction to classical complex analysis, New York-London, 1979.

[5] B. Buffoni, E. N. Dancer and J. F. Toland, The regularity and local bifurcation of steady periodic water waves, Arch. Ration. Mech. Anal. 152 (2000), 207-240.

[6] B. Buffoni, E. N. Dancer and J. F. Toland, The sub-harmonic bifurcation of Stokes waves, Arch. Ration. Mech. Anal. 152 (2000), 241-271.

[7] B. Buffoni and J. F. Toland, Analytic theory of global bifurcation, Princeton University Press, Princeton and Oxford, 2003.

[8] A. Constantin, The trajectories of particles in Stokes waves, Invent. Math. 166 (2006), 523-535.

[9] A. Constantin, M. Ehrnström and E. Wahlén, Symmetry of steady periodic gravity water waves with vorticity, Duke Math. J. 140 (2007), 591-603.

[10] A. Constantin and J. Escher, Symmetry of steady periodic surface water waves with vorticity, J. Fluid Mech. 498 (2004), 171-181.

[11] A. Constantin and J. Escher, Analyticity of periodic traveling free surface water waves with vorticity, preprint (2009).

[12] A. Constantin and W. Strauss, Exact steady periodic water waves with vorticity, Comm. Pure Appl. Math. 57 (2004), 481-527.

[13] A. Constantin and W. Strauss, Rotational steady water waves near stagnation, Phil. Trans. Roy. Soc. London A 365 (2007), 2227-2239.

[14] A. Constantin and W. Strauss, Pressure beneath a Stokes wave, Comm. Pure Appl. Math. 53 (2010), 533-557.

[15] M. G. Crandall and P. H. Rabinowitz, Bifurcation from simple eigenvalues, J. Funct. Anal. 8 (1971), 321-340.

[16] A. F. T. da Silva and D. H. Peregrine, Steep, steady surface waves on water of finite depth with constant vorticity, J. Fluid Mech. 195 (1988), 281-302.

[17] H. Dym and H. P. McKean, Fourier series and integrals, Academic Press, New York-London, 1972.

[18] D. Gilbarg and N. S. Trudinger, Elliptic partial differential equations of second order, SpringerVerlag, Berlin, 2001.

[19] I. G Jonsson, Wave-current interactions, in The Sea, pp. 65-120, Wiley, New York, 1990.

[20] G. Keady and J. Norbury, On the existence theory for irrotational water waves, Math. Proc. Cambridge Philos. Soc. 83 (1978), 137-157.

[21] D. Kinderlehrer, L. Nirenberg and J. Spruck, Regularity in elliptic free boundary problems, $J$. Analyse Math. 34 (1978), 86-119.

[22] J. Ko and W. Strauss, Effect of vorticity on steady water waves, J. Fluid Mech. 608 (2008), $197-215$. 
[23] J. Ko and W. Strauss, Large-amplitude steady rotational water waves, Eur. J. Mech. B Fluids 27 (2008), 96-109.

[24] P. Koosis, Introduction to $H_{p}$-spaces, 2nd edition, Cambridge University Press, Cambridge-New York, 1998.

[25] H. Lewy, A note on harmonic functions and a hydrodynamical application, Proc. Amer. Math. Soc. 3 (1952), 111-113.

[26] Z. Nehari, Conformal mapping, McGraw-Hill Book Co., Inc., New York, Toronto, London, 1952.

[27] H. Okamoto and M. Shoji, The mathematical theory of permanent progressive water waves, World Scientific, New Jersey-London-Singapore-Hong Kong, 2001.

[28] A. K. Pichler-Tennenberg, On an equation related to Stokes waves, Ph.D. thesis, University of Bath (United Kingdom), 2002.

[29] Ch. Pommerenke, Boundary behaviour of conformal maps, Springer-Verlag, Berlin-HeidelbergNew York, 1992.

[30] W. Rudin, Real and complex analysis, McGraw-Hill Book Co., New York, 1987.

[31] E. Shargorodsky and J. F. Toland, Bernoulli free-boundary problems, Mem. Amer. Math. Soc. 196 (2008), no. 914.

[32] E. M. Stein, Harmonic analysis: real-variable methods, orthogonality, and oscillatory integrals, Princeton University Press, Princeton, NJ, 1993.

[33] C. Swan, I. P. Cummins and R. L. James, An experimental study of two-dimensional surface water waves propagating on depth-varying currents, J. Fluid Mech. 428 (2001), 273-304.

[34] W. Thomson (Lord Kelvin), On a disturbing infinity in Lord Rayleigh's solution for waves in a plane vortex stratum, Nature 23 (1880), 45-46.

[35] P. D. Thompson, The propagation of small surface disturbances through rotational flow, Ann. N.Y. Acad. Sci. 51 (1949), 463-474.

[36] J. F. Toland, Stokes waves, Topol. Methods Nonlinear Anal. 7 (1996), 1-48.

[37] J. F. Toland, On a pseudo-differential equation for Stokes waves, Arch. Ration. Mech. Anal. 162 (2002), 179-189.

[38] A. Torchinsky, Real-variable methods in harmonic analysis, Dover Publications, Inc., Mineola, NY, 2004.

[39] E. Varvaruca, Singularities of Bernoulli free boundaries, Comm. Partial Differential Equations 31 (2006), 1451-1477.

[40] E. Varvaruca, Some geometric and analytic properties of solutions of Bernoulli free-boundary problems, Interfaces Free Bound. 9 (2007), 367-381.

[41] E. Varvaruca, Bernoulli free-boundary problems in strip-like domains and a property of permanent waves on water of finite depth, Proc. Roy. Soc. Edinburgh A 138 (2008), 1345-1362.

[42] E. Varvaruca, On some properties of traveling water waves with vorticity, SIAM J. Math. Anal. 39 (2008), 1686-1692.

[43] E. Varvaruca, On the existence of extreme waves and the Stokes conjecture with vorticity, $J$. Differential Equations 246 (2009), 4043-4076.

[44] E. Wahlén, Steady water waves with a critical layer, J. Differential Equations 246 (2009), 24682483.

[45] T. Yanagi, Coastal Oceanography, Kluwer Academic Publishers, Dordrecht, 2003. 